\title{
Muon Track Reconstruction and Data Selection Techniques in AMANDA
}

\author{
The AMANDA Collaboration
}

J. Ahrens ${ }^{11}$, X. Bai ${ }^{1}$, R. Bay ${ }^{9}$, S.W. Barwick ${ }^{10}$, T. Becka ${ }^{11}$, J.K. Becker ${ }^{2}$, K.-H. Becker ${ }^{2}$, E. Bernardini ${ }^{4}$, D. Bertrand ${ }^{3}$, A. Biron ${ }^{4}$, D.J. Boersma ${ }^{4}$, S. Böser ${ }^{4}$, O. Botner ${ }^{17}$, A. Bouchta ${ }^{17}$, O. Bouhali ${ }^{3}$, T. Burgess ${ }^{18}$, S. Carius ${ }^{6}$, T. Castermans ${ }^{13}$, D. Chirkin ${ }^{9}$, B. Collin ${ }^{8}$, J. Conrad ${ }^{17}$, J. Cooley ${ }^{15}$, D.F. Cowen ${ }^{8}$, A. Davour ${ }^{17}$, C. De Clercq ${ }^{19}$, T. DeYoung ${ }^{12}$, P. Desiati ${ }^{15}$, J.-P. Dewulf ${ }^{3}$, P. Ekström ${ }^{2}$, T. Feser ${ }^{11}$, M. Gaug ${ }^{4}$, T.K. Gaisser ${ }^{1}$, R. Ganugapati ${ }^{15}$, H. Geenen ${ }^{2}$, L. Gerhardt ${ }^{10}$, A. Groß ${ }^{2}$, A. Goldschmidt ${ }^{7}$, A. Hallgren ${ }^{17}$, F. Halzen ${ }^{15}$, K. Hanson ${ }^{15}$, R. Hardtke ${ }^{15}$, T. Harenberg ${ }^{2}$, T. Hauschildt ${ }^{4}$, K. Helbing ${ }^{7}$, M. Hellwig ${ }^{11}$, P. Herquet ${ }^{13}$, G.C. Hill ${ }^{15}$, D. Hubert ${ }^{19}$, B. Hughey ${ }^{15}$, P.O. Hulth ${ }^{18}$, K. Hultqvist ${ }^{18}$, S. Hundertmark ${ }^{18}$, J. Jacobsen ${ }^{7}$, A. Karle ${ }^{15}$, M. Kestel $^{8}$, L. Köpke ${ }^{11}$, M. Kowalski ${ }^{4}$, K. Kuehn ${ }^{10}$, J.I. Lamoureux ${ }^{7}$, H. Leich ${ }^{4}$, M. Leuthold ${ }^{4}$, P. Lindahl ${ }^{6}$, I. Liubarsky ${ }^{5}$, J. Madsen ${ }^{16}$, P. Marciniewski ${ }^{17}$, H.S. Matis ${ }^{7}$, C.P. McParland ${ }^{7}$, T. Messarius ${ }^{2}$, Y. Minaeva ${ }^{18}$, P. Miočinović ${ }^{9}$, P. C. Mock ${ }^{10}$,

R. Morse ${ }^{15}$, K.S. Münich ${ }^{2}$, J. Nam ${ }^{10}$, R. Nahnhauer ${ }^{4}$, T. Neunhöffer ${ }^{11}$, P. Niessen ${ }^{19}$, D.R. Nygren ${ }^{7}$, H. Ögelman ${ }^{15}$, Ph. Olbrechts ${ }^{19}$,

C. Pérez de los $\operatorname{Heros}^{17}$, A.C. Pohl ${ }^{18}$, R.Porrata ${ }^{10}$, P.B. Price ${ }^{9}$, G.T. Przybylski ${ }^{7}$, K. Rawlins ${ }^{15}$, E. Resconi ${ }^{4}$, W. Rhode ${ }^{2}$, M. Ribordy ${ }^{13}$, S. Richter ${ }^{15}$, J. Rodríguez Martino ${ }^{18}$, D. Ross ${ }^{10}$, H.-G. Sander ${ }^{11}$, K. Schinarakis ${ }^{2}$, S. Schlenstedt ${ }^{4}$, T. Schmidt ${ }^{4}$, D. Schneider ${ }^{15}$, R. Schwarz ${ }^{15}$, A. Silvestri ${ }^{10}$, M. Solarz ${ }^{9}$, G.M. Spiczak ${ }^{16}$, C. Spiering ${ }^{4}$, M. Stamatikos ${ }^{15}$, D. Steele ${ }^{15}$, P. Steffen ${ }^{4}$, R.G. Stokstad ${ }^{7}$, K.-H. Sulanke ${ }^{4}$, O. Streicher ${ }^{4}$, I. Taboada ${ }^{14}$, L. Thollander ${ }^{18}$,

S. Tilav ${ }^{1}$, W. Wagner ${ }^{2}$, C. Walck ${ }^{18}$, Y.-R. Wang ${ }^{15}$, C.H. Wiebusch ${ }^{2}$,

C. Wiedemann ${ }^{18}$, R. Wischnewski ${ }^{4}$, H. Wissing ${ }^{4}$, K. Woschnagg ${ }^{9}$, G. Yodh $^{10}$

(1) Bartol Research Institute, University of Delaware, Newark, DE 19716, USA

(2) Fachbereich 8 Physik, BU Wuppertal, D-42097 Wuppertal, Germany

(3) Université Libre de Bruxelles, Science Faculty, Brussels, Belgium (4) DESY-Zeuthen, D-15738 Zeuthen, Germany

(5) Blackett Laboratory, Imperial College, London SW7 2BW, UK

(6) Dept. of Technology, Kalmar University, S-39182 Kalmar, Sweden

(7) Lawrence Berkeley National Laboratory, Berkeley, CA 94720, USA

(8) Dept. of Physics, Pennsylvania State University, University Park, PA 16802, USA

(9) Dept. of Physics, University of California, Berkeley, CA 94720, USA

(10) Dept. of Physics and Astronomy, Univ. of California, Irvine, CA 92697, USA

(11) Institute of Physics, University of Mainz, D-55099 Mainz, Germany

ऋ Corresponding author. Address: Fachbereich 8 Physik, Gaussstraße 20, BU Wuppertal, D42097 Wuppertal, Germany. Phone : +49 (0)202 439 3531. Fax : +49 (0)202 439 2662. Email: Wiebusch@physik.uni-wuppertal.de 
(12) Dept. of Physics, University of Maryland, College Park, MD 20742, USA

(13) University of Mons-Hainaut, 7000 Mons, Belgium

(14) Dept. de Física, Universidad Simón Bolivar, Caracas, 1080, Venezuela

(15) Dept. of Physics, University of Wisconsin, Madison, WI 53706, USA

(16) Physics Dept., University of Wisconsin, River Falls, WI 54022, USA

(17) Div. of High Energy Physics, Uppsala University, S-75121 Uppsala, Sweden

(18) Dept. of Physics, Stockholm University, SE-10691 Stockholm, Sweden

(19) Vrije Universiteit Brussel, Dienst ELEM, B-1050 Brussels, Belgium

\begin{abstract}
The Antarctic Muon And Neutrino Detector Array (AMANDA) is a high-energy neutrino telescope operating at the geographic South Pole. It is a lattice of photomultiplier tubes buried deep in the polar ice between $1500 \mathrm{~m}$ and $2000 \mathrm{~m}$. The primary goal of this detector is to discover astrophysical sources of high energy neutrinos. A high-energy muon neutrino coming through the earth from the Northern Hemisphere can be identified by the secondary muon moving upward through the detector.

The muon tracks are reconstructed with a maximum likelihood method. It models the arrival times and amplitudes of Cherenkov photons registered by the photomultipliers. This paper describes the different methods of reconstruction, which have been successfully implemented within AMANDA. Strategies for optimizing the reconstruction performance and rejecting background are presented. For a typical analysis procedure the direction of tracks are reconstructed with about $2^{\circ}$ accuracy.
\end{abstract}

Key words: AMANDA, track reconstruction, neutrino telescope, neutrino astrophysics

PACS: 95.55.Vj, 95.75.Pq, 29.40.Ka, 29.85.+c

\title{
1 Introduction
}

The Antarctic Muon And Neutrino Detector Array [1], AMANDA, is a large volume neutrino detector at the geographic South Pole. It is a lattice of photo-multiplier tubes (PMTs) buried deep in the optically transparent polar ice. The primary goal of this detector is to detect high-energy neutrinos from astrophysical sources, and determine their arrival time, direction and energy. When a high-energy neutrino interacts in the polar ice via a charged current reaction with a nucleon $N$ :

$$
\nu_{\ell}+N \rightarrow \ell+X
$$

it creates a hadronic cascade, $X$, and a lepton, $\ell=e, \mu, \tau$. These particles generate Che-

renkov photons, which are detected by the PMTs. Each lepton flavor generates a different 
signal in the detector. The two basic detection modes are sketched in figure 1.

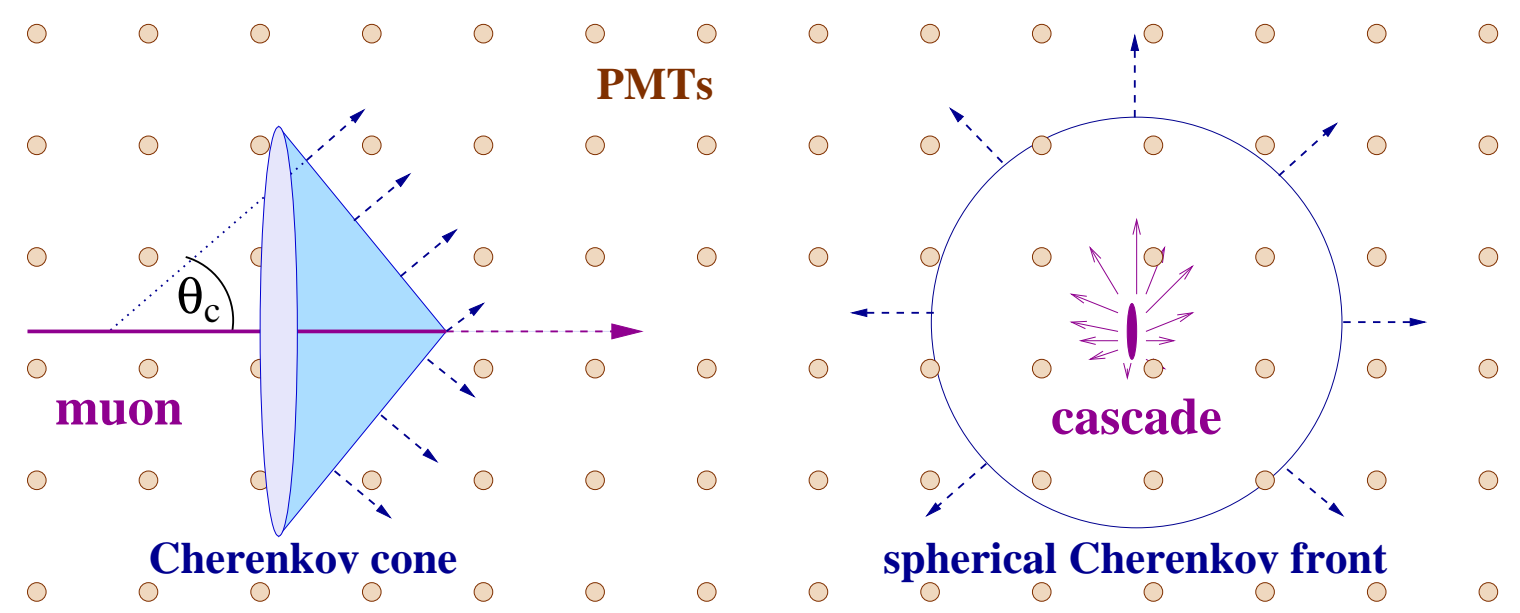

Figure 1. Detection modes of the AMANDA detector: Left: muon tracks induced by muon-neutrinos; Right: Cascades from electron- or tau-neutrinos.

A high-energy $\nu_{\mu}$ charged current interaction creates a muon, which is nearly collinear with the neutrino direction; having a mean deviation angle of $\psi=0.7^{\circ} \times\left(E_{\nu} / \mathrm{TeV}\right)^{-0.7}[2]$, which implies an accuracy requirement of $\lesssim 1^{\circ}$ for reconstructing the muon direction.

The high-energy muon emits a cone of Cherenkov light at a fixed angle $\theta_{\mathrm{c}}$. It is determined by $\cos \theta_{\mathrm{c}}=(n \cdot \beta)^{-1}$, where $n \simeq 1.32$ is the index of refraction in the ice. For relativistic particles, $\beta \simeq 1$, and $\theta_{\mathrm{c}} \approx 41^{\circ}$. The direction of the muon is reconstructed from the time and amplitude information of the PMTs illuminated by the Cherenkov cone.

Radiative energy loss processes generate secondary charged particles along the muon trajectory, which also produce Cherenkov radiation. These additional photons allow an estimate of the muon energy. However, the resolution is limited by fluctuations of these processes. This estimate is a lower bound on the neutrino energy, because it is based on the muon energy at the detector. The interaction vertex may be far outside the detector.

The $\nu_{e}$ and $\nu_{\tau}$ channels are different. The electron from a $\nu_{e}$ will generate an electromagnetic cascade, which is confined to a volume of a few cubic meters. This cascade coincides with the hadronic cascade $X$ of the primary interaction vertex. The optical signature is an expanding spherical shell of Cherenkov photons with a larger intensity in the forward direction. The tau from a $\nu_{\tau}$ will decay immediately and also generate a cascade. However, at energies $>1 \mathrm{PeV}$ this cascade and the vertex are separated by several tens of meters, connected by a single track. This signature of two extremely bright cascades is unique for high-energy $\nu_{\tau}$, and it is called a double bang event [3].

The measurement of cascade-like events is restricted to interactions close to the detector, thus requiring larger instrumented volumes than for $\nu_{\mu}$ detection. Also the accuracy of the direction measurement is worse for cascades than for long muon tracks. However, when 
the flux is diffuse, the $\nu_{e}$ and $\nu_{\tau}$ channels also have clear advantages. The backgrounds from atmospheric neutrinos are smaller. The energy resolution is significantly better since the full energy is deposited in or near the detector. The cascade channel is sensitive to all neutrino flavors because the neutral current interactions also generate cascades. In this paper we focus on the reconstruction of muon tracks; details on the reconstruction of cascades are described in [4].

The most abundant events in AMANDA are atmospheric muons, created by cosmic rays interacting with the Earth's atmosphere. At the depth of AMANDA their rate exceeds the rate of muons from atmospheric neutrinos by five orders of magnitude. Since these muons are absorbed by the earth, a muon track from the lower hemisphere is a unique signature for a neutrino-induced muon ${ }^{2}$. The reconstruction procedure must have good angular resolution, good efficiency, and allow excellent rejection of down-going atmospheric muons.

This paper describes the methods used to reconstruct muon tracks recorded in the AMANDA experiment. The AMANDA-II detector is introduced in section 2. The reconstruction algorithms and their implementation are described in sections 3 to 5 . Section 6 summarizes event classes for which the reconstruction may fail and strategies to identify and eliminate such events. The performance of the reconstruction procedure is shown in section 7 . We discuss possible improvements in section 8 .

\section{The AMANDA Detector}

The AMANDA-II detector (see figure 2) has been operating since January 2000 with 677 optical modules $(\mathrm{OM})$ attached to 19 strings. Most of the OMs are located between $1500 \mathrm{~m}$ and $2000 \mathrm{~m}$ below the surface. Each OM is a glass pressure vessel, which contains an 8inch hemispherical PMT and its electronics. AMANDA-B10 ${ }^{3}$, the inner core of $302 \mathrm{OMs}$ on 10 strings, has been operating since 1997.

One unique feature of AMANDA is that it continuously measures atmospheric muons in coincidence with the South Pole Air Shower Experiment surface arrays SPASE-1 and SPASE-2 [7]. These muons are used to survey the detector and calibrate the angular resolution (see section 7 and $[8,9]$ ), while providing SPASE with additional information for cosmic ray composition studies [10].

The PMT signals are processed in a counting room at the surface of the ice. The analog signals are amplified and sent to a majority logic trigger [11]. There the pulses are discrim-

2 Muon neutrinos above $1 \mathrm{PeV}$ are absorbed by the Earth. At these ultra-high-energies (UHE), however, the the muon background from cosmic rays is small and UHE muons coming from the horizon and above are most likely created by UHE neutrinos. The search for these UHE neutrinos is described in $[5,6]$.

3 Occasionally in the paper we will refer to this earlier detector instead of the full AMANDA-II detector. 


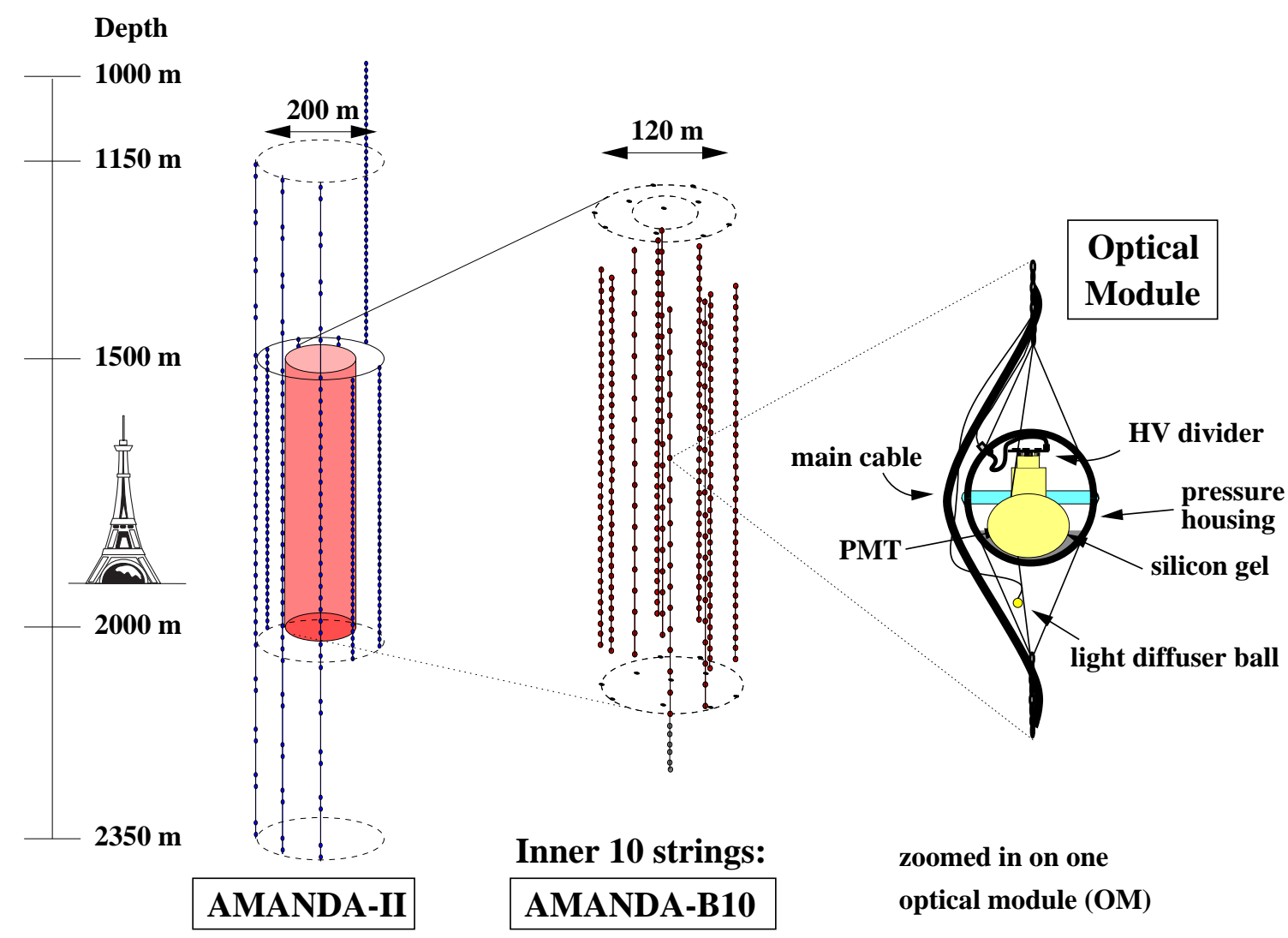

Figure 2. The AMANDA-II detector. The scale is illustrated by the Eiffel tower at the left.

inated and a trigger is formed if a minimum number of hit PMTs are observed within a time window of typically $2 \mu \mathrm{s}$. Typical trigger thresholds were 16 hit PMT for AMANDAB10 and 24 for AMANDA-II. For each trigger the detector records the peak amplitude and up to 16 leading and trailing edge times for each discriminated signal. The time resolution achieved after calibration is $\sigma_{t} \simeq 5 \mathrm{~ns}$ for the PMTs from the first 10 strings, which are read out via coaxial or twisted pair cables. For the remaining PMTs, which are read out with optical fibers the resolution is $\sigma_{t} \simeq 3.5 \mathrm{~ns}$. In the cold environment of the deep ice the PMTs have low noise rates of typically $1 \mathrm{kHz}$.

The timing and amplitude calibration, the array geometry, and the optical properties of the ice are determined by illuminating the array with known optical pulses from in situ sources [11]. Time offsets are also determined from the response to through-going atmospheric muons [12].

The optical absorption length in the ice is typically $110 \mathrm{~m}$ at $400 \mathrm{~nm}$ with a strong wavelength dependence. The effective scattering length at $400 \mathrm{~nm}$ is on average $\simeq 20 \mathrm{~m}$. It is defined as $\lambda_{\mathrm{s}} /\left(1-\left\langle\cos \theta_{\mathrm{s}}\right\rangle\right)$, where $\lambda_{\mathrm{s}}$ is the scattering length and $\theta_{\mathrm{s}}$ is the scattering angle. The ice parameters vary strongly with depth due to horizontal ice layers, i.e., variations in the concentration of impurities which reflect past geological events and climate changes [13-19]. 


\section{Reconstruction Algorithms}

The muon track reconstruction algorithm is a maximum likelihood procedure. Prior to reconstruction simple pattern recognition algorithms, discussed in section 4 , generate the initial estimates required by the maximum likelihood reconstructions.

\subsection{Likelihood Description}

The reconstruction of an event can be generalized to the problem of estimating a set of unknown parameters $\{\mathbf{a}\}$, e.g. track parameters, given a set of experimentally measured values $\{\mathbf{x}\}$. The parameters, $\{\mathbf{a}\}$, are determined by maximizing the likelihood $\mathcal{L}(\mathbf{x} \mid \mathbf{a})$ which for independent components $x_{i}$ of $\mathbf{x}$ reduces to

$$
\mathcal{L}(\mathbf{x} \mid \mathbf{a})=\prod_{i} p\left(x_{i} \mid \mathbf{a}\right)
$$

where $p\left(x_{i} \mid \mathbf{a}\right)$ is the probability density function (p.d.f.) of observing the measured value $x_{i}$ for given values of the parameters $\{\mathbf{a}\}[20]$.

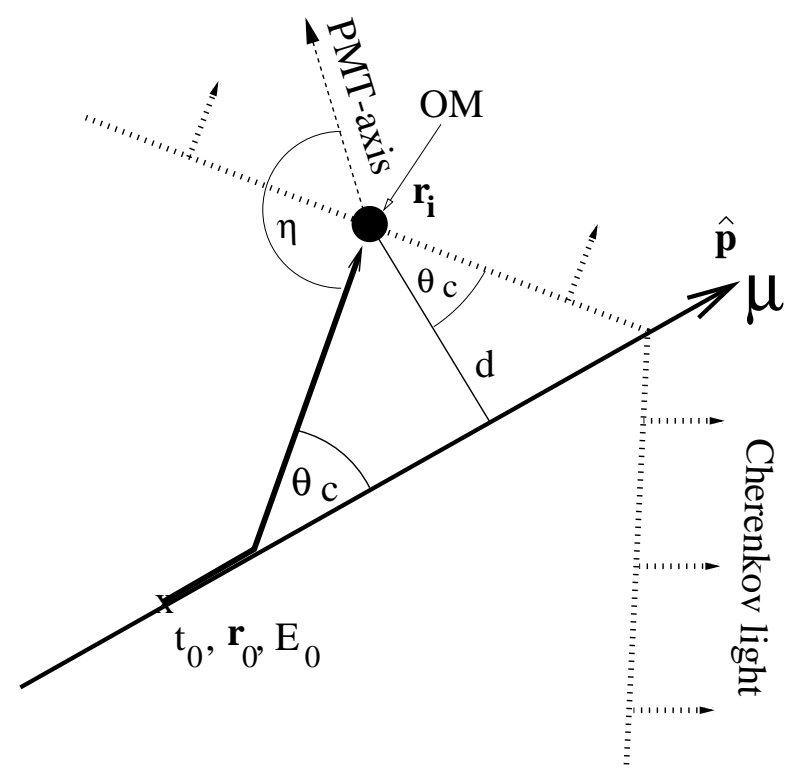

Figure 3. Cherenkov light front: definition of variables

To simplify the discussion we assume that the Cherenkov radiation is generated by a single infinitely long muon track (with $\beta=1$ ) and forms a cone. It is described by the following parameters:

$$
\mathbf{a}=\left(\mathbf{r}_{\mathbf{0}}, t_{0}, \hat{\mathbf{p}}, E_{0}\right)
$$

and illustrated in figure 3 . Here, $\mathbf{r}_{\mathbf{0}}$ is an arbitrary point on the track. At time $t_{0}$, the muon passes $\mathbf{r}_{\mathbf{0}}$ with energy $E_{0}$ along a direction $\hat{\mathbf{p}}$. The geometrical coordinates contain five degrees of freedom. Along this track, Cherenkov photons are emitted at a fixed angle 
$\theta_{\text {c }}$ relative to $\hat{\mathbf{p}}$. Within the reconstruction algorithm it is possible to use a different coordinate system, e.g. $\mathbf{a}=(d, \eta, \ldots)$. The reconstruction is performed by minimizing $-\log (\mathcal{L})$ with respect to $\mathbf{a}$.

The values $\{\mathbf{x}\}$ presently recorded by AMANDA are the time $t_{i}$ and duration TOT $_{i}$ (Time Over Threshold) of each PMT signal, as well as the peak amplitude $A_{i}$ of the largest pulse in each PMT. PMTs with no signal above threshold are also accounted for in the likelihood function. The hit times give the most relevant information. Therefore we will first concentrate on $p(t \mid \mathbf{a})$.

\subsubsection{Time Likelihood}

According to the geometry in figure 3, photons are expected to arrive at OM $i$ (at $\left.\mathbf{r}_{\mathbf{i}}\right)$ at time

$$
t_{\text {geo }}=t_{0}+\frac{\hat{\mathbf{p}} \cdot\left(\mathbf{r}_{\mathbf{i}}-\mathbf{r}_{\mathbf{0}}\right)+d \tan \theta_{\mathrm{c}}}{c_{\mathrm{vac}}},
$$

with $c_{\mathrm{vac}}$ the vacuum speed of light ${ }^{4}$. It is convenient to define a relative arrival time, or time residual

$$
t_{\text {res }} \equiv t_{\text {hit }}-t_{\text {geo }}
$$

which is the difference between the observed hit time and the hit time expected for a "direct photon", a Cherenkov photon that travels undelayed directly from the muon to an OM without scattering.
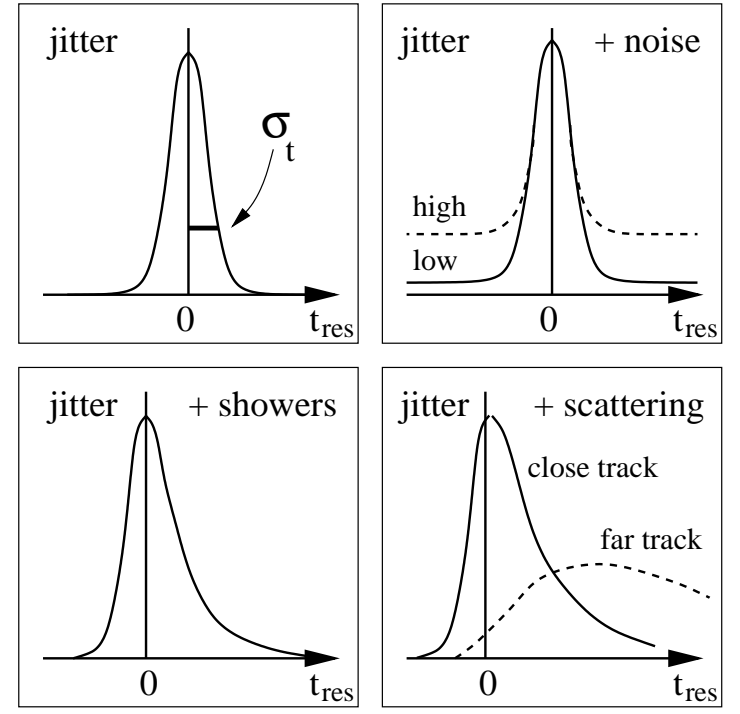

Figure 4. Schematic distributions of arrival times $t_{\text {tes }}$ for different cases: Top left: PMT jitter. Top right: the effect of jitter and random noise. Bottom left: The effect of jitter and secondary cascades along the muon track. Bottom right: The effect of jitter and scattering.

4 We note that equation 4 neglects the effect that Cherenkov light propagates with group velocity as pointed out in [21]. It was shown in [14] that for AMANDA this approximation is justified. 
In the ideal case, the distribution, $p\left(t_{\text {res }} \mid \mathbf{a}\right)$, would be a delta function. However, in a realistic experimental situation this distribution is broadened and distorted by several effects, which are illustrated in figure 4 . The PMT jitter limits the timing resolution $\sigma_{t}$. Noise, e.g. dark noise of the PMT, leads to additional hits which are random in time. These effects can generate negative $t_{\text {res }}$ values, which would mimic unphysical causality violations. Secondary radiative energy losses along the muon trajectory create photons that arrive after the ideal Cherenkov cone. These processes are stochastic, and their relative photon yield fluctuates.

In AMANDA, the dominant effect on photon arrival times is scattering in the ice ${ }^{5}$. The effect of scattering depends strongly on the distance, $d$, of the OM from the track as illustrated in figure 4 . Since the PMTs have a non-uniform angular response, $p\left(t_{\text {res }}\right)$ also depends on the orientation, $\eta$, of the OM relative to the muon track (see figure 3). OMs facing away from the track can only see light that scatters back towards the PMT face. On average this effect shifts $t_{\text {res }}$ to later times and modifies the probability of a hit.

The simplest time likelihood function is based on a likelihood constructed from $p_{1}$, the p.d.f. for arrival times of single photons $i$ at the locations of the hit OMs

$$
\mathcal{L}_{\text {time }}=\prod_{i=1}^{N_{\text {hits }}} p_{1}\left(t_{\mathrm{res}, i} \mid \mathbf{a}=d_{i}, \eta_{i}, \ldots\right)
$$

Note that one OM may contribute to this product with several hits. The function $p_{1}\left(t_{\text {res }, i} \mid \mathbf{a}\right)$ is obtained from the simulation of photon propagation through ice (see section 3.2). However, this description is limited, because the electrical and optical signal channels can only resolve multiple photons separated by a few $100 \mathrm{~ns}$ and $\simeq 10 \mathrm{~ns}$, respectively. Within this time window, only the arrival time of the first pulse is recorded.

This first photon is usually less scattered than the average single photon, which modifies the probability distribution of the detected hit time. The arrival time distribution of the first of $N$ photons is given by

$$
p_{N}^{1}\left(t_{\text {res }}\right)=N \cdot p_{1}\left(t_{\text {res }}\right) \cdot\left(\int_{t_{\text {res }}}^{\infty} p_{1}(t) d t\right)^{(N-1)}=N \cdot p_{1}\left(t_{\text {tres }}\right) \cdot\left(1-P_{1}\left(t_{\text {res }}\right)\right)^{(N-1)}
$$

$P_{1}$ is the cumulative distribution of the single photon p.d.f.. The function $p_{N}^{1}\left(t_{\text {res }}\right)$ is called the multi-photo-electron $(M P E)$ p.d.f. and correspondingly defines $\mathcal{L}_{\mathrm{MPE}}$.

This concept can be extended to the more general case of $p_{N}^{k}\left(t_{\text {res }}\right)$, the p.d.f. for the $k^{\text {th }}$ photon out of a total of $N$ to arrive at $t_{\text {res }}$, given by

$$
p_{N}^{k}\left(t_{\mathrm{res}}\right)=N \cdot\left(\begin{array}{c}
N-1 \\
k-1
\end{array}\right) \cdot p_{1}\left(t_{\mathrm{res}}\right) \cdot\left(1-P_{1}\left(t_{\mathrm{res}}\right)\right)^{(N-k)} \cdot\left(P_{1}\left(t_{\mathrm{res}}\right)\right)^{(k-1)}
$$

$p_{N}^{k}\left(t_{\text {res }}\right)$ specifies the likelihood of arrival times of individual photoelectrons for averaged

${ }^{5}$ In water detectors this effect is neglected [22] or treated as a small correction [23]. 
time series of $N$ photoelectrons. With waveform recording the arrival times and amplitudes of individual pulses can be resolved.

When the number of photoelectrons, $N$, is not measured precisely enough, multi-photon information can be included via another method. Instead of measuring $N$, the p.d.f. of the first photoelectron can be calculated by convolving the MPE p.d.f. $p_{N}^{1}(t, d)$ with the Poisson probability $P_{N}^{\text {Poisson }}(\mu)$, where $\mu$ is the mean expected number of photoelectrons as a function of the distance, $d$.

$$
p_{\mu}^{1}\left(t_{\mathrm{res}}\right)=\frac{1}{N} \sum_{i=1}^{\infty} \frac{\mu^{i} e^{-\mu}}{i !} \cdot p_{i}^{1}\left(t_{\mathrm{res}}\right)=\frac{\mu}{1-e^{-\mu}} \cdot p_{1}\left(t_{\mathrm{res}}\right) \cdot e^{-\mu P_{1}\left(t_{\mathrm{res}}\right)}
$$

This result is called the Poisson Saturated Amplitude (PSA) p.d.f. [24, 25] and correspondingly defines $\mathcal{L}_{\mathrm{PSA}}$. The constant $N=1-e^{-\mu}$ renormalizes the p.d.f. to unity.

The probability of (uncorrelated) noise hits is small. They are further suppressed by a hit cleaning procedure (section 5.3), which is applied before reconstruction. They are included in the likelihood function by simply adding a constant p.d.f. $p_{0}$.

\subsubsection{Hit and No-Hit Likelihood}

The likelihood in the previous section relies only on the measured arrival times of photons. However, the topology of the hits is also important. PMTs with no hits near a hypothetical track or PMTs with hits far from the track are unlikely.

A likelihood utilizing this information can be constructed as

$$
\mathcal{L}_{\text {hit }}=\prod_{i=1}^{N_{\mathrm{ch}}} P^{\mathrm{hit}, \mathrm{i}} \cdot \prod_{i=N_{\mathrm{ch}}+1}^{N_{\mathrm{OM}}} P^{\mathrm{no}-\mathrm{hit}, \mathrm{i}}
$$

where $N_{\mathrm{ch}}$ is the number of hit OMs and $N_{\mathrm{OM}}$ the number of operational OMs. The probabilities $P^{\text {hit }}$ and $P^{\text {no-hit }}$ of observing or not observing a hit depend on the track parameters a. Additional hits due to random noise are easily incorporated: $P^{\text {no-hit }} \rightarrow$ $\tilde{P}^{\text {no-hit }} \equiv P^{\text {no-hit }} \cdot P^{\text {no-noise }}$ and $P^{\text {hit }} \rightarrow \tilde{P}^{\text {hit }}=1-\tilde{P}^{\text {no-hit }}$.

Assuming that the probability $P_{1}^{\text {hit }}$ is known for a single photon, the hit and no-hit probabilities of OMs for $n$ photons can be calculated:

$$
P_{n}^{\text {no-hit }}=\left(1-P_{1}^{\text {hit }}\right)^{n}
$$

and

$$
\begin{aligned}
P_{n}^{\text {hit }} & =1-P_{n}^{\text {no-hit }} \\
& =1-\left(1-P_{1}^{\text {hit }}\right)^{n}
\end{aligned}
$$

The number of photons, $n$, depends on $E_{\mu}$, the energy of the muon: $n=n\left(E_{\mu}\right)$. For a 
fixed track geometry, the likelihood (equation 10) can be used to reconstruct the muon energy.

\subsubsection{Amplitude Likelihood}

The peak amplitudes recorded by AMANDA can be fully incorporated in the likelihood [26], which is particularly useful for energy reconstruction. The likelihood can be written as

$$
\mathcal{L}=\frac{W}{N_{\mathrm{OM}}} \cdot \prod_{i=1}^{N_{\mathrm{OM}}} w_{i} \cdot P_{i}\left(A_{i}\right)
$$

where $P_{i}\left(A_{i}\right)$ is the probability that OM $i$ observes an amplitude $A_{i}$, with $A_{i}=0$ for unhit OMs. $W$ and $w_{i}$ are weight factors, which describe deviations of the individual OM and the total number of hit OMs from the expectation. $P_{i}$ depends on the mean number $\mu$ of expected photoelectrons:

$$
P_{i}\left(A_{i}\right)=P^{\text {hit }} \cdot\left(1-P_{i}^{\text {th }}\right) \cdot \frac{P\left(A_{i}, \mu\right)}{P\left(\left\langle A_{i}\right\rangle, \mu\right)} .
$$

The probability $P_{i}\left(A_{i}\right)$ is normalized to the probability of observing the most likely amplitude $\left\langle A_{i}\right\rangle . P_{i}^{\text {th }}(\mu)$ is the probability that a signal of $\mu$ does not produce a pulse amplitude above the discriminator threshold. As before, $P^{\text {hit }}=1-P^{\text {no-hit }}$, where the no-hit probability is given by Poisson statistics: $P^{\text {no-hit }}=\exp (-\mu) \cdot\left(1-P^{\text {noise }}\right)$. The probability of $A_{i}=0$ is a special case: $P_{i}(0)=P^{\text {no-hit }}+P^{\text {hit }} \cdot P_{i}^{\text {th }}$. Energy reconstructions based on this formulation of the likelihood will be referred to as Full $E_{\text {reco }}$.

An alternative energy reconstruction technique (see section 3.2.4) uses a neural net which is fed with energy sensitive parameters.

\subsubsection{Zenith Weighted (Bayesian) Likelihood}

Another extension of the likelihood [27-29] incorporates external information about the muon flux via Bayes' Theorem. This theorem states that for two hypotheses $A$ and $B$,

$$
P(A \mid B)=\frac{P(B \mid A) P(A)}{P(B)} .
$$

Identifying $A$ with the track parameters a and $B$ with the observations x, equation 14 gives the probability that the inferred muon track a was in fact responsible for the observed event $\mathbf{x} \cdot P(\mathbf{x} \mid \mathbf{a})$ is the probability that $\mathbf{a}$, assumed to be true, would generate the event $\mathbf{x}$ - in other words, the likelihood described in the previous sections. $P(\mathbf{a})$ is the prior probability of observing the track a; i.e., the relative frequencies of different muon tracks as a function of their parameters. $P(\mathbf{x})$, which is independent of the track parameters $\mathbf{a}$, is a normalization constant which ensures that equation 14 defines a proper probability. 
Because the likelihood is only defined up to an arbitrary constant factor, this normalization may be ignored in the present context.

In order to obtain $P(\mathbf{a} \mid \mathbf{x})$, one thus has to determine the prior probability distribution, $P(\mathbf{a})$, of how likely the various possible track directions are a priori. The reconstruction maximizes the product of the p.d.f. and the prior.

The flux of muons deep underground is reasonably well known from previous experiments. Any point source of muons would be at most a small perturbation on the flux of penetrating atmospheric muons and muons created by atmospheric neutrinos. The most striking feature of the background flux from atmospheric muons is the strong dependence on zenith angle. For vertically down-going tracks it exceeds the flux from neutrino induced muons by about 5 orders of magnitude but becomes negligible for up-going tracks. This dependence, which is modeled by a Monte Carlo calculation [30], acts as a zenith dependent weight to the different muon hypotheses, a. With this particular choice, some tracks, which would otherwise reconstruct as up-going, reconstruct as down-going tracks. This greatly reduces the rate at which penetrating atmospheric muons are mis-reconstructed as up-going neutrino events [31]. In principle, a more accurate prior could be used. It would need to include the depth and energy dependence of the atmospheric muons as well as the angular dependence of atmospheric neutrino induced muons.

Upon completion of this work, we learned that this technique was developed independently by the NEVOD neutrino detector collaboration [32] who were able to extract an atmospheric neutrino from a background of $10^{10}$ atmospheric muons in a small $\left(6 \times 6 \times 7.5 \mathrm{~m}^{3}\right)$ surface detector.

\subsubsection{Combined Likelihoods}

The likelihood function $\mathcal{L}_{\text {time }}$ of the hit times is the most important for track reconstruction. However, it is useful to include other information like the hit probabilities. The combined p.d.f. from Equations 7 and 10 is

$$
\mathcal{L}_{\mathrm{MPE} \oplus \mathrm{Phit}^{\mathrm{P} \text { no-hit }}}=\mathcal{L}_{\mathrm{MPE}} \cdot\left(\mathcal{L}_{\text {hit }}\right)^{w}
$$

which is particularly effective. Here $w$ is an optional weight factor which allows the adjustment of the relative weight of the two likelihoods. This likelihood is sensitive not only to the track geometry but also to the energy of the muon.

As discussed in section 3.1.4, the zenith angle dependent prior function, $P(\theta)$, can be included as a multiplicative factor. This combination

$$
\mathcal{L}_{\text {Bayes }}=P(\theta) \cdot \mathcal{L}_{\text {time }}
$$

has been used in the analysis of atmospheric neutrinos [30]. However, all of these improved likelihoods are limited by the underlying model assumption of a single muon track. 


\subsection{Likelihood Implementation}

The actual implementation of the likelihoods requires detailed knowledge of the photon propagation in the ice. On the other hand, efficiency considerations and numeric problems favor a simple and robust method.

The photon hit probabilities and arrival time distributions are simulated as functions of all relevant parameters with a dedicated Monte Carlo simulation and archived in large look-up tables. This simulation is described in [26, 30, 33].

The AMANDA Collaboration has followed different strategies for incorporating this data into the reconstruction. In principle the probability density functions are taken directly from these archives. However, one has to face several technical difficulties due to the memory requirements of the archived tables, as well as numeric problems related to the normalization of interpolated bins and the calculation of multi-photon likelihoods.

Alternatively, one can simplify the model and parametrize these archives with analytical functions, which depend only on a reduced set of parameters. Comparisons of two independent parametrizations $[24,34]$ show that the direct and parametrized approaches yield similar results in terms of efficiency. This indicates that the parametrization itself is not limiting the reconstruction quality; rather, as mentioned earlier, the reconstruction is limited by the assumptions of the model being fit. Therefore, we will concentrate on only one parametrization.

\subsubsection{Analytical Parametrization}

A simple parametrization of the arrival time distributions can be achieved with the following function, which we call Pandel function. It is a gamma distribution and its usage is motivated by an analysis of laser light signals in the BAIKAL experiment [35]. There, it was found that for the case of an isotropic, monochromatic and point-like light source, $p_{1}\left(t_{\mathrm{res}}\right)$ can be expressed in the form

$$
\begin{aligned}
p\left(t_{\mathrm{res}}\right) & \equiv \frac{1}{N(d)} \frac{\tau^{-(d / \lambda)} \cdot t_{\mathrm{res}}^{(d / \lambda-1)}}{\Gamma(d / \lambda)} \cdot e^{-\left(t_{\mathrm{res}} \cdot\left(\frac{1}{\tau}+\frac{c_{\text {medium }}}{\lambda_{a}}\right)+\frac{d}{\lambda_{a}}\right)} \\
N(d) & =e^{-d / \lambda_{a}} \cdot\left(1+\frac{\tau \cdot c_{\text {medium }}}{\lambda_{a}}\right)^{-d / \lambda}
\end{aligned}
$$

without special assumptions on the actual optical parameters. Here, $c_{\text {medium }}=c_{\mathrm{vac}} / n$ is the speed of light in ice, $\lambda_{a}$ the absorption length, $\Gamma(d / \lambda)$ the Gamma function and $N(d)$ a normalization factor, which is given by equation 18 . This formulation has free parameters $\lambda$ and $\tau$, which are unspecified functions of the distance $d$ and the other geometrical parameters. They are empirically determined by a Monte Carlo model. 
The Pandel function has some convenient mathematical properties: it is normalized, it is easy to compute, and it can be integrated analytically over the time, $t_{\text {res }}$, which simplifies the construction of the multi-photon (MPE) time p.d.f.. For small distances the function has a pole at $t=0$ corresponding to a high probability of an unscattered photon. Going to larger values of $d$, longer delay times become more likely. For distances larger than the critical value $d=\lambda$, the power index to $t_{\text {res }}$ changes sign, reflecting that the probability of undelayed photons vanishes: essentially all photons are delayed due to scattering.

The large freedom in the choice of the two parameter functions $\tau$ (units of time) and $\lambda$ (units of length) and the overall reasonable behavior is the motivation to use this function to parametrize not only the time p.d.f. for point-like sources, but also for muon tracks [34]. The Pandel function is fit to the distributions of delay times for fixed distances $d$ and angles $\eta$ (between the PMT axis and the Cherenkov cone). These distributions are previously obtained from a detailed photon propagation Monte Carlo for the Cherenkov light from muons. The free fit parameters are $\tau, \lambda, \lambda_{a}$ and the effective distance $d_{\text {eff }}$, which will be introduced next.
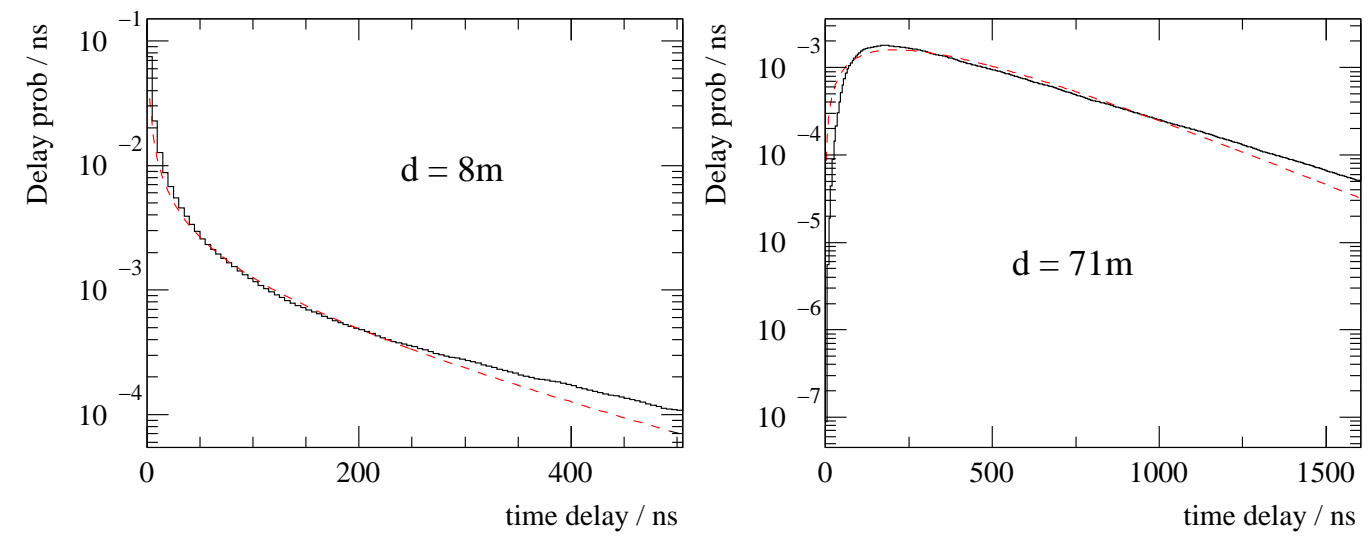

Figure 5. Comparison of the parametrized Pandel function (dashed curves) with the detailed simulation (black histograms) at two distances $d$ from the muon track.

When investigating the fit results as a function of $d$ and angle $\eta$ (see figure 3 ), we observe that already for a simple ansatz of constant $\tau, \lambda$ and $\lambda_{a}$ the optical properties in AMANDA are described sufficiently well within typical distances. The dependence on $\eta$ is described by an effective distance $d_{\text {eff }}$ which replaces $d$ in equation 17 . This means that the time delay distributions for backward illumination of the PMT is found to be similar to a head-on illumination at a larger distance. The following parameters are obtained for a specific ice model, and are currently used in the reconstruction:

$$
\begin{array}{ll}
\tau=557 \mathrm{~ns} & d_{\mathrm{eff}}=a_{0}+a_{1} \cdot d \\
\lambda=33.3 \mathrm{~m} & a_{1}=0.84 \\
\lambda_{a}=98 \mathrm{~m} & a_{0}=3.1 \mathrm{~m}-3.9 \mathrm{~m} \cdot \cos (\eta)+4.6 \mathrm{~m} \cdot \cos ^{2}(\eta)
\end{array}
$$

A comparison of the results from this parametrization with the full simulation is shown in figure 5 for two extreme distances. The simple approximation describes the behavior of the full simulation reasonably well. However, this simple overall description has a limited accuracy, especially for $d \approx \lambda$ (not shown). Reconstructions, based on the Pandel function 
with different ice models, and a generic reconstruction, that uses the full simulation results, yield similar results. These comparisons indicate that the results of the reconstruction do not critically depend on the fine tuning of the underlying ice models, and it justifies the use of the above simple model.

\subsubsection{Extension to realistic PMT signals}

Although the Pandel function is the basis of a simple normalized likelihood, it has several deficiencies. It is not defined for negative $t_{\text {res }}$, it ignores PMT jitter, and it has a pole at $t_{\text {res }}=0$, which causes numerical difficulties. These problems can be resolved by convolving the Pandel function, equation 17, with a Gaussian, which accounts for the PMT jitter. Unfortunately such convolution requires significant computing time.

Instead the Pandel function is modified by extending it to negative times, $t_{\text {res }}<0$, with a (half) Gaussian of width $\sigma_{g}$. The effects of PMT jitter are only relevant for small values of $t_{\text {res }}$. For times $t_{\text {res }} \geq t_{1}$ the original function is used, and the two parts are connected by a spline interpolation (3rd order polynomial). The result, $\hat{P}\left(t_{\mathrm{res}}\right)$, is called upandel function.

Using $t_{1}=\sqrt{2 \pi} \cdot \sigma_{g}$ and requiring further a smooth interpolation and the normalization to be unchanged, the polynomial coefficients $a_{j}$ and normalization of the Gaussian $N_{g}$ can be calculated analytically [34]. The free parameter $\sigma_{g}$ includes all timing uncertainties, not just the PMT jitter. Good reconstruction results are achieved for a large range $10 \mathrm{~ns} \leq$ $\sigma_{g} \leq 20 \mathrm{~ns}$.

\subsection{3 $P^{\text {hit }} P^{\text {no-hit }}$ Parametrization}

The normalization $N(d)$ in equation 18 is used to construct a hit probability function, $P_{\text {hit }}$. The function $P_{n}^{\text {hit }}$ with $P_{1}^{\text {hit }} \equiv N(d)$, is fit to the hit probability determined by the full AMANDA detector simulation, as a function of distance, orientation and muon energy. The free parameters are the Pandel parameters $\tau$ and $\lambda, \lambda_{a}, \hat{d}$ and $\tilde{n}$. The effective distance $\hat{d}$, is similar to the effective distance in the Pandel parametrization. We define $\tilde{n}$ as the power index of equation 11, which corresponds to an effective number of photons. It is important to understand that $N(d)$ is not a hit probability and $\tilde{n}$ is not just a number of photons. They are constructs, that are calibrated with a Monte Carlo simulation. Technically, the power index, $\tilde{n} \equiv N$ in equation 11 , factorizes into $\tilde{n}\left(\eta, E_{\mu}\right)=\epsilon\left(\eta, E_{\mu}\right) \cdot n\left(E_{\mu}\right)$. The variable $n$, where $n=n(E)$, is related to the number of photons incident on the PMT and its absolute efficiency. The factor $\epsilon\left(\eta, E_{\mu}\right)$ is related to the orientation dependent PMT sensitivity but also accounts for the energy dependent angular emission profile of photons with respect to the bare muon. 


\subsubsection{Energy Reconstruction}

The reconstruction of the track geometry is a search for five parameters. If the muon energy is added as a fit parameter, the minimization is significantly slower. Therefore, the energy reconstruction is performed in two steps. First, the track geometry is reconstructed without the energy parameter. Then these geometric parameters are used in an energy reconstruction, that only determines the energy. However, if the time likelihood utilizes amplitude information, e.g. in the combined likelihood, equation 7 , or the PSA likelihood, equation 9, the track parameters also depend on the energy. In this case the energy and geometric parameters must be reconstructed together. Currently, three different approaches are used to reconstruct the muon energy. They are compared in section 7.3.

(1) The simplest method utilizes the $P^{\text {hit }} P^{\text {no-hit }}$ reconstruction (see sections 3.1 .2 and 3.2.3).

(2) The Full $E_{\text {reco }}$ method (see section 3.1.3) models the measured amplitudes in a likelihood for reconstructing the energy. This algorithm performs better, but it is more dependent on the quality of the amplitude calibration of the OMs.

(3) An alternative way to measure the energy is based on a neural network [36]. The neural network uses 6-6-3-1 and 6-3-5-1 feed-forward architecture for AMANDA-B10 and AMANDA-II, respectively. The energy correlated variables which are used as input are the mean of the measured amplitudes (ADC), the mean and RMS of the arrival times (LE) or pulse durations (TOT), the total number of signals, the number of OMs hit and the number of OMs with exactly one hit.

Less challenging than a full reconstruction, a lower energy threshold is determined by requiring a minimum number of hit OMs. The number of hit OMs is correlated with the energy of the muon. Since celestial neutrinos are believed to have a substantially harder spectrum than atmospheric neutrinos, an excess of high multiplicity events would indicate that a hard celestial source exists. Values for this parameter determined from AMANDA data already set a tight upper limit on the diffuse flux of high-energy celestial neutrinos [37].

\subsubsection{Cascade Reconstruction}

The reconstruction of cascade like events is described in detail elsewhere [4]. The basic approach is similar to the track reconstruction. It assumes events form a point light source with photons propagating spherically outside with a higher intensity in the forward direction. The cascade reconstruction also uses the Pandel function (see equation 17) with parameters that are specific for cascades.

In several muon analyses, a cascade fit is used as a competing model. In cases where the cascade fit achieves a better likelihood than the track reconstruction, the track hypothesis is rejected. In particular this is used as a selection criterion to reject background events which are mis-reconstructed due to bright secondary cascades. 


\section{First Guess Pattern Recognition}

The likelihood reconstructions need an initial track hypothesis to start the minimization. The initial track is derived from first guess methods, which are fast analytic algorithms that do not require an initial track.

\subsection{Direct Walk}

A very efficient first guess method is the direct walk algorithm. It is a pattern recognition algorithm based on carefully selected hits, which were most likely caused by direct photons.

The four step procedure starts by selecting track elements, the straight line between any two hit OMs at distance $d$, which are hit with a time difference

$$
|\Delta t|<\frac{d}{c_{\text {vac }}}+30 \text { ns with } d>50 \mathrm{~m} .
$$

The known positions of the OMs define the track element direction $(\theta, \phi)$. The vertex position $(x, y, z)$ is taken at the center between the two OMs. The time at the vertex $t_{0}$ is defined as the average of the two hit times.

In a next step, the number of associated hits (AH) are calculated for each track element. Associated hits are those with $-30 \mathrm{~ns}<t_{\mathrm{res}}<300 \mathrm{~ns}$ and $d<25 \mathrm{~m} \cdot\left(t_{\mathrm{res}}+30\right)^{1 / 4}(t$ in ns), where $d$ is the distance between hit OM and track element and $t_{\text {res }}$ is the time residual, which is defined in Equation 5. After selecting these associated hits, track elements of poor quality are rejected by requiring: $N_{\mathrm{AH}} \geq 10$ and $\sigma_{L} \equiv \sqrt{\left(\frac{1}{N_{\mathrm{AH}}} \sum_{i}\left(L_{i}-\langle L\rangle\right)^{2}\right)} \geq 20 \mathrm{~m}$. Here, the "lever arm" $L_{i}$ is the distance between the vertex of the track element and the point on the track element which is closest to OM $i$ and $\langle L\rangle$ is the average of all $L_{i}$-values. Track elements that fulfill these criteria qualify as track candidates (TC).

Frequently, more than one track candidate is found. In this case, a cluster search is performed for all track candidates that fulfill the quality criterion:

$$
\begin{aligned}
& Q_{\mathrm{TC}} \geq 0.7 \cdot Q_{\max }, \quad \text { where } \\
& Q_{\max }=\max \left(Q_{\mathrm{TC}}\right) \quad \text { and } \\
& Q_{\mathrm{TC}}=\min \left(N_{\mathrm{AH}}, 0.3 \mathrm{~m}^{-1} \cdot \sigma_{L}+7\right) .
\end{aligned}
$$

In the cluster search, the "neighbors" of each track candidate are counted, where neighbors are track candidates with space angle differences of less than $15^{\circ}$. The cluster with the largest number of track candidates is selected. 
In the final step, the average direction of all track candidates inside the cluster defines the initial track direction. The track vertex and time are taken from the central track candidate in the cluster. Well separated clusters can be used to identify independent muon tracks in events which contain multiple muons (see section 6.1).

\subsection{Line-Fit}

The line-fit [38] algorithm produces an initial track on the basis of the hit times with an optional amplitude weight. It ignores the geometry of the Cherenkov cone and the optical properties of the medium and assumes light traveling with a velocity $\mathbf{v}$ along a 1-dimensional path through the detector. The locations of each PMT, $\mathbf{r}_{i}$, which are hit at a time $t_{i}$ can be connected by a line:

$$
\mathbf{r}_{i} \approx \mathbf{r}+\mathbf{v} \cdot t_{i}
$$

A $\chi^{2}$ to be minimized is defined as:

$$
\chi^{2} \equiv \sum_{i=1}^{N_{\mathrm{hit}}}\left(\mathbf{r}_{i}-\mathbf{r}-\mathbf{v} \cdot t_{i}\right)^{2}
$$

where $N_{\text {hit }}$ is the number of hits. The $\chi^{2}$ is minimized by differentiation with respect to the free fit parameters $\mathbf{r}$ and $\mathbf{v}$. This can be solved analytically:

$$
\mathbf{r}=\left\langle\mathbf{r}_{i}\right\rangle-\mathbf{v} \cdot\left\langle t_{i}\right\rangle \quad \text { and } \quad \mathbf{v}=\frac{\left\langle\mathbf{r}_{i} \cdot t_{i}\right\rangle-\left\langle\mathbf{r}_{i}\right\rangle \cdot\left\langle t_{i}\right\rangle}{\left\langle t_{i}^{2}\right\rangle-\left\langle t_{i}\right\rangle^{2}}
$$

where $\left\langle x_{i}\right\rangle \equiv \frac{1}{N_{\text {hit }}} \sum_{i}^{N_{\text {hit }}} x_{i}$ denotes the mean of parameter $x$ with respect to all hits.

The line-fit thus yields a vertex point $\mathbf{r}$, and a direction $\mathbf{e}=\mathbf{v}_{\mathrm{LF}} /\left|\mathbf{v}_{\mathrm{LF}}\right|$. The zenith angle is given by $\theta_{\mathrm{LF}} \equiv-\arccos \left(v_{z} /\left|\mathbf{v}_{\mathrm{LF}}\right|\right)$.

The time residuals (equation 5) for this initial track generally do not follow the distribution expected for a Cherenkov model. If the $t_{0}$ parameter of the initial track is shifted to better agree with a Cherenkov model, subsequent reconstructions converge better (see section 5.2.3).

The absolute speed $v_{\mathrm{LF}} \equiv|\mathbf{v}|$, of the line-fit is the mean speed of the light propagating through the 1-dimensional detector projection. Spherical events (cascades) and high energy muons have low $v_{\mathrm{LF}}$ values, and thin, long events (minimally ionizing muon tracks) have large values. 


\subsection{Dipole Algorithm}

The dipole algorithm considers the unit vector from one hit OM to the subsequently hit $\mathrm{OM}$ as an individual dipole moment. Averaging over all individual dipole moments yields the global moment $\mathbf{M}$. It is calculated in two steps. First, all hits are sorted according to their hit times. Then a dipole-moment $\mathbf{M}$ is calculated:

$$
\mathbf{M} \equiv \frac{1}{N_{\mathrm{ch}}-1} \cdot \sum_{i=2}^{N_{\mathrm{ch}}} \frac{\mathbf{r}_{i}-\mathbf{r}_{i-1}}{\left|\mathbf{r}_{i}-\mathbf{r}_{i-1}\right|}
$$

It can be expressed via an absolute value $M_{\mathrm{DA}} \equiv|\mathbf{M}|$ and two angles $\theta_{\mathrm{DA}}$ and $\phi_{\mathrm{DA}}$. These angles define the initial track.

The dipole algorithm does not generate as good an initial track as the direct walk or the line-fit, but it is less vulnerable to a specific class of background events: almost coincident atmospheric muons from independent air showers in which the first muon hits the bottom and the second muon hits the top of the detector.

\subsection{Inertia Tensor Algorithm}

The inertia tensor algorithm is based on a mechanical picture. The pulse amplitude from a PMT at $\mathbf{r}_{i}$ corresponds to a virtual mass $a_{i}$ at $\mathbf{r}_{i}$. One can then define the tensor of inertia I of that virtual mass distribution. The origin is the center of gravity (COG) of the mass distribution. The COG-coordinates and the tensor of inertia components are given by:

$$
\begin{aligned}
\mathbf{C O G} & \equiv \sum_{i=1}^{N_{\mathrm{ch}}}\left(a_{i}\right)^{w} \cdot \mathbf{r}_{i} \quad \text { and } \\
I^{k, l} & \equiv \sum_{i=1}^{N_{\mathrm{ch}}}\left(a_{i}\right)^{w} \cdot\left[\delta^{k l} \cdot\left(\mathbf{r}_{i}\right)^{2}-r_{i}^{k} \cdot r_{i}^{l}\right] .
\end{aligned}
$$

The amplitude weight $w \geq 0$ can be chosen arbitrarily. The most common settings are $w=0$ (ignoring the amplitudes) and $w=1$ (setting the virtual masses equal to the amplitudes). The tensor of inertia has three eigenvalues $I_{j}, j \in\{1,2,3\}$, corresponding to its three main axes $\mathbf{e}_{j}$. The smallest eigenvalue $I_{1}$ corresponds to the longest axis $\mathbf{e}_{1}$. In the case of a long track-like event $I_{1} \ll\left\{I_{2}, I_{3}\right\}$ and $\mathbf{e}_{1}$ approximates the direction of the track. The ambiguity in the direction along the $e_{1}$ axis is resolved by choosing the direction where the average $\mathrm{OM}$ hit time is latest. In the case of a cascade-like event, $I_{1} \approx I_{2} \approx I_{3}$. The ratios between the $I_{j}$ can be used to determine the sphericity of the event. 


\section{Aspects of the technical Implementation}

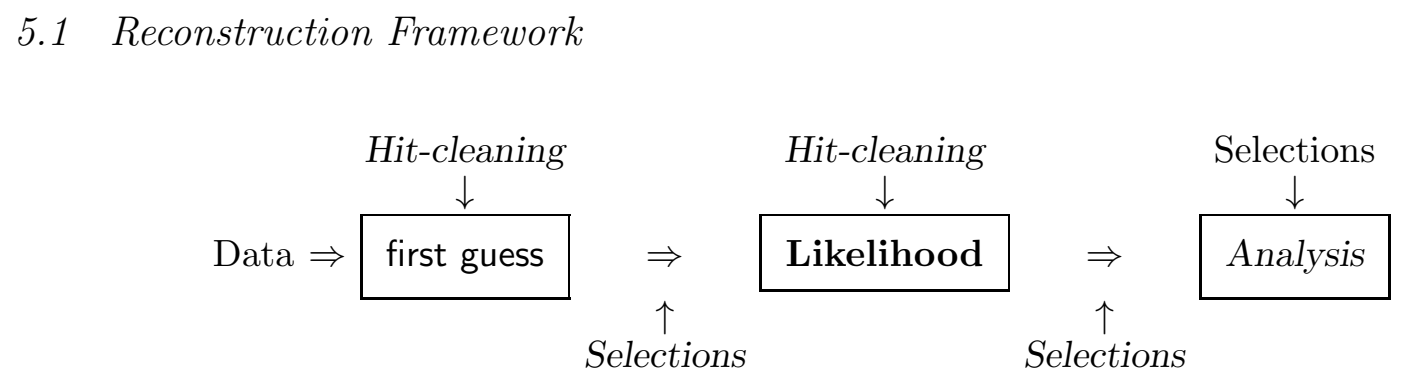

Figure 6. Schematic principle of the reconstruction chain

The basic reconstruction procedure, sketched in figure 6, is sequential. A fast reconstruction program calculates the initial track hypothesis for the likelihood reconstruction. All reconstruction programs may use a reduced set of hits in order to suppress noise hits and other detector artifacts. Event selection criteria can be applied after each step to reduce the event sample, and allow more time consuming calculations at later reconstruction stages. This procedure may iterate with more sophisticated but slower algorithms analyzing previous results. The final step is usually the production of Data Summary Tape (DST) like information, usually in form of PAW N-tuples [39]. A detailed description of this procedure can be found in [40].

The reconstruction framework is implemented with the recoos program [41], which is based on the $r d m c$ library [42] and the SiEGMuND software package [43]. The recoos program is highly modular, which allows flexibility in the choice and combination of algorithms.

\subsection{Likelihood Maximization}

The aim of the reconstruction is to find the track hypothesis which corresponds to the maximum likelihood. This is done by minimizing $-\log (\mathcal{L})$ with respect to the track parameters. We have implemented several minimization procedures.

The likelihood space for AMANDA events is often characterized by several minima. Local likelihood minima can arise due to symmetries in the detector, especially in the azimuth angle, or due to unexpected hit times caused by scattering. In the example, shown in figure 7 , the reconstruction converged on a local minimum, because of non-optimal starting values. Several techniques, which are used to find the global minimum, are here presented. In particular, the iterative reconstruction, section 5.2.2, solves the problem and converges to the global minimum. One generally assumes that the global minimum corresponds to the true solution, but this is not always correct due to stochastic nature of light emission and detection. Such events cannot be reconstructed properly and have to be rejected using quality parameters (see section 6.2). 


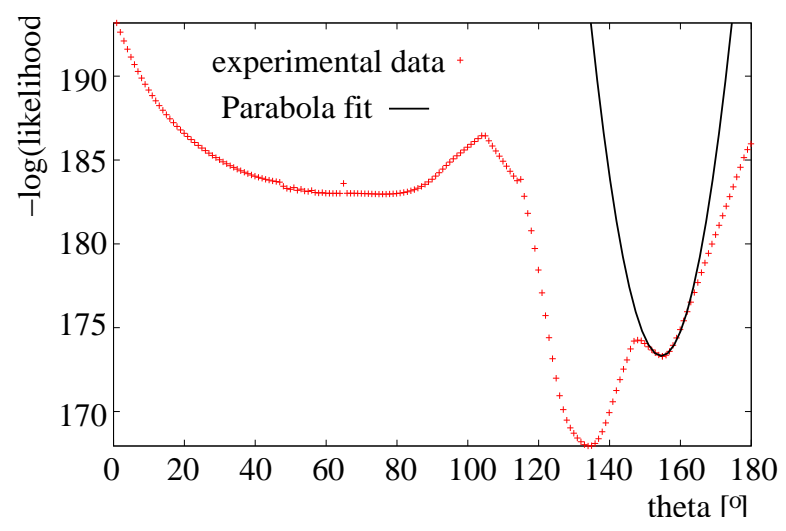

Figure 7. An example of the likelihood space (1-dimensional projection) for a specific AMANDA event. Shown is $-\log (\mathcal{L})$ as function of the zenith angle. Each point represents a fit, for which the zenith angle was fixed and the other track parameters were allowed to vary in order to find the best minimum. A local minimum which was found by a gradient likelihood minimization is indicated by a fitted parabola. Improved methods that avoid this are described in the text.

\subsubsection{Minimization algorithms}

The reconstruction framework allows us to use and compare these numerical minimization algorithms: Simplex [44], Powell's [44], Minuit [45] (using the minimize method), and Simulated annealing [44]. The Simplex algorithm is the fastest algorithm. Powell's method and Minuit are $\sim 5$ times slower than the Simplex algorithm. The reconstruction results from Minuit and the Simplex algorithm are nearly identical and almost as good as the Powell results. Exceptions occur in less than $1 \%$ of the cases, when these methods fail and stop at the extreme zenith angles $\theta=0^{\circ}$ and $\theta=180^{\circ}$. The Simulated annealing algorithm is less sensitive to local minima than the other algorithms, but it is much slower and requires fine-tuning.

\subsubsection{Iterative Reconstruction}

The iterative reconstruction algorithm successfully copes with the problem of local minima and extreme zenith angles by performing multiple reconstructions of the same event. Each reconstruction starts with a different initial track. Therefore, the fast Simplex algorithm is sufficient.

The ability to find the global minimum depends strongly on the quality of the initial track. A systematic scan of the full parameter space for initial seeds is not feasible. Instead the iterative algorithm concentrates on the direction angles, zenith and azimuth, and uses reasonable values for the spatial coordinates. The following procedure yields good results.

The result of a first minimization is saved as a reference. Then both direction angles are randomly selected. The track point, $\mathbf{r}_{\mathbf{0}}$, is transformed to the point on the new track, which is closest to the center of gravity of hits. The time, $t_{0}$, of this point is shifted to match the Cherenkov expectation (see section 5.2.3). Then a new minimization is started. 
If the minimum is less than the reference minimum, it is saved as the new reference. This procedure is iterated $n$ times, and the best minima found for zenith angles above and below the horizon, are saved, and used to generate an important selection parameter (see section 6.2).

This algorithm substantially reduces the number of false minima found, after a few iterations. For $n=6$ roughly $95 \%$ of the results are in the vicinity of the asymptotic optimum for $n \rightarrow \infty$. For $n \simeq 20$ more than $99 \%$ of the results are the global minimum. Despite the fast convergence, the iterative reconstruction requires significant CPU time, which limits its use to reduced data sets.

\subsubsection{Lateral shift and time residual}

The efficiency of finding the global minimum of the likelihood function can be improved by translating the arbitrary vertex and/or time origin of the output track from the first guess algorithm before application of the full maximum likelihood method.

Transformation of $\mathbf{r}_{\mathbf{0}}$. In general this "vertex" point is arbitrary in the infinite track approximation used, and first guess methods may produce positions distant from the detector. During the likelihood minimization, numerical errors can be avoided and the convergence improved by shifting this point along the direction of the track towards the point closest to the center of gravity of hits (see equation 26). The vertex time $t_{0}$ is transformed accordingly: $\Delta\left(t_{0}\right)=\Delta\left(\mathbf{r}_{\mathbf{0}}\right) / c$.

Transformation of $t_{0}$. The time $t_{0}$ obtained from first guess algorithms is not calculated from a full Cherenkov model. The efficiency of the likelihood reconstruction can be improved by shifting the $t_{0}$ such that the time residuals, equation 5 , fit better to a Cherenkov model. In particular it is useful to avoid negative $t_{\text {res }}$, which would correspond to causal-

ity violations. This can be achieved by transforming $t_{0} \rightarrow t_{0}-t_{\text {res }}^{-}$, where $t_{\text {res }}^{-}$is the most negative time residual.

\subsubsection{Coordinates and restricted parameters}

The track coordinates a, which are used by the likelihood, are independent of the coordinates actually chosen for the minimization. Therefore, the coordinate system can be chosen arbitrarily. Any of the parameters in this coordinate system can be kept fixed. During the minimization parameterization functions translate the coordinates as necessary. The most commonly used coordinates are $\mathbf{r}_{\mathbf{0}}$ and the zenith and azimuth angles $\theta$, $\phi$.

The freedom in the choice of coordinates can be used to improve the numerical minimization, for systematic studies, or to fix certain parameters according to external knowledge. An example is the reconstruction of coincident events with the SPASE surface arrays [8]. Here, we fix the location of the trajectory to coincide with the core location at the surface 
as measured by SPASE. Then, the direction is determined with the AMANDA reconstruction subject to this constraint.

Under certain circumstances the allowed range of the reconstruction parameters is restricted. The most important example here is to restrict the reconstructed zenith angle to above or below the horizon, to find the most likely up- or down-going tracks, respectively. Comparing the quality of the two solutions can be used for background rejection. Technically the constrained fit is accomplished by multiplying the likelihood by a prior, which is zero outside the allowed parameter range.

\subsection{Preprocessing and Hit cleaning}

The data must be filtered and calibrated before reconstruction. Defective OMs are removed, and the amplitudes and hit times are calibrated. A hit cleaning procedure identifies and flags hits which appear to be noise or electronic effects, such as cross talk or after-pulsing. These hits are not used in the reconstruction, but they are retained for post-reconstruction analysis.

The hit cleaning procedure can be based on simple and robust algorithms, because the PMTs have low noise rates. Noise and after-pulse hits are strongly suppressed by rejecting hits that are isolated in time and space from other signals in the detector. Typically a hit is considered to be noise if there is no hit within a distance of $60 \mathrm{~m}$ to $100 \mathrm{~m}$ and a time of $\pm 300 \mathrm{~ns}$ to $\pm 600 \mathrm{~ns}$. Cross talk hits are identified by examining the amplitudes and pulse widths of the individual pulses and by analyzing the correlations of uncalibrated hit times with hits of large amplitude in channel combinations which are known to cross talk to each other. The required cross talk correlation map was determined independently in a dedicated calibration campaign.

\subsection{Processing Speeds}

The first guess algorithms are sufficiently fast that the execution time is dominated by file input/output and the software framework. Typical fit times are $\approx 20 \mathrm{~ms}$ per event on a $850 \mathrm{MHz}$ Pentium-III Linux PC. The processing speed of the likelihood reconstructions can vary significantly depending on the number of free parameters, the number of iterations, the minimization algorithm, and the experimental parameters like the number of hit OMs. These effects dominate the differences in processing speeds due to different reconstruction

algorithms. The typical execution time for a 16-fold iterative likelihood reconstructions using the simplex minimizer to reconstruct the 5 free track parameters is $\simeq 250 \mathrm{~ms}$ per event. 


\section{Background Rejection}

The performance of the reconstruction depends strongly on the quality and background selection criteria. The major classes of background events in AMANDA (see section 6.1) are suppressed by the quality parameters presented in section 6.2. Optimization strategies for the selection criteria are summarized in section 6.3. Finally, we evaluate the reconstruction performance in section 7 .

\subsection{Background Classes}

Most background events from atmospheric muons are well reconstructed and can be rejected by selecting up-going reconstructed events. However, there is a small fraction of mis-reconstructed events, amounting to about $10^{-2}$ for the unbiased and about $10^{-4}$ for the zenith-weighted reconstruction. These events are rejected by additional selection criteria described in section 6.2. These background events are classified as follows.

Nearly horizontal muons: These events have true incident angles close to the horizon. A small error in the reconstruction causes them to appear as up-going. These events are not severely mis-reconstructed, but occur due to the finite angular resolution.

Muon bundles: The spatial separation between multiple muons from a single air shower, a muon bundle, is usually small enough that the event can be described by a single bright muon track. If the separation is too large, the reconstruction fails.

Cascades: Bright stochastic energy losses (e.g. bremsstrahlung) produce additional light, which distorts the Cherenkov cone from the muon. Cascades emit most of their light with the same Cherenkov angle as the muon, but some light is emitted at other angles. These secondary events can cause the reconstruction to fail, especially when the cascade(s) produce more light than the muon itself. A special class of these events are muons which pass outside the detector and release a bright cascade, which can mimic an up-going hit pattern.

Stopping muons: Over the depth of the detector the muon flux changes by a factor of $\sim 2$, since muons lose their energy and stop. These muons can create an up-going hit pattern, especially when the muon stops just before entering the detector from the side.

Scattering layers: The scattering of light in the polar ice cap varies with depth. Light from bright events, can mimic an up-going hit pattern, in particular when it traverses layers of higher scattering.

Corner clippers: These are events where the muon passes diagonally below the detector. The light travel upwards through the detector mimicking an up-going muon.

Uncorrelated coincident muons: Due to the large size of the AMANDA detector, the probability of muons from two independent air showers forming a single event is small on the trigger level but not negligible. If an initial muon traverses the bottom of the detector and a later muon traverses the top, the combination can be reconstructed as an up-going muon. 
Electronic artifacts: Noise, cross talk and other transient electronic malfunctions are generally small effects, but they can occasionally produce hits, which distort the time pattern. Such effects become important after a selection process of several orders of magnitude.

\subsection{Quality Parameters}

Background events, which pass a zenith angle selection, need to be rejected by applying selection criteria on quality parameters. These parameters usually evaluate information, which is not optimally exploited in the reconstruction. The detailed choice of quality parameters is specific to each analysis. Here, we summarize the most important categories.

The number of direct hits, $N_{\text {dir }}\left(t_{1}: t_{2}\right)$, is the number of hits with small time residuals: $t_{1}<t_{\mathrm{res}}<t_{2}$ (see equation 5). Un-scattered photons provide the best information for the reconstruction, and a large number of $N_{\text {dir }}$ indicates high quality information in the event. Empirically reasonable values are $t_{1} \simeq-15 \mathrm{~ns}$ and $t_{2}$ between $=+25 \mathrm{~ns}$ and $+150 \mathrm{~ns}$, depending on the specific analysis.

The length of the event $L$ is obtained by projecting each hit OM onto the reconstructed track and taking the distance between the two outermost of these points. $L$ can be considered as the "lever arm" of the reconstruction. Larger values corresponding to a more robust and precise reconstruction of the track's direction. This parameter is particularly powerful when calculated for direct hits only, and is then referred to as $L_{\text {dir }}\left(t_{1}: t_{2}\right)$. Length requirements are efficient against corner clippers, stopping muons and cascades.

The absolute value of the likelihood at the maximum is a good parameter to evaluate the quality of a reconstruction. Here, a useful observable is the likelihood parameter $\mathrm{L}$ which is defined as

$$
\mathrm{L} \equiv-\frac{\log (\mathcal{L})}{N_{\text {free }}}
$$

where $N_{\text {free }}$ is the degrees of freedom (e.g. $N_{\text {free }}=N_{\text {hits }}-5$ for a track reconstruction). For Gaussian probability distributions this expression corresponds to the reduced chi-square. $\mathrm{L}$ can be used as a selection parameter, smaller values corresponding to higher quality. A selection of events with good $\mathrm{L}^{P^{\text {hit }} P^{\text {no-hit }}}$ values is efficient against stopping muons.

Comparing $\mathrm{L}$ from different reconstructions is a powerful technique. Cascade-like events will have a better likelihood from a cascade reconstruction than one from a track reconstruction.

Another efficient rejection method is to compare $L$ for the best up-going versus the best down-going reconstruction of a single event. If the up-going reconstruction is not significantly better than the down-going reconstruction, the event is rejected. These values can be obtained from the iterative reconstruction method (section 5.2.2) or by restricting the 
parameter space. This method is particularly powerful when the down-going reconstruction uses a zenith weighted likelihood (section 3.1.4).

The reconstruction methods consider the p.d.f. for each hit separately but ignore correlations. Therefore, the reconstructions assign the same likelihood to tracks where all hits cluster at one end of the reconstructed track and tracks where the same number of hits are smoothly distributed along the track. The latter hit pattern indicates a successful track reconstruction, while the former hit pattern may be caused by a background event. The smoothness parameter $S$ was inspired by the Kolmogorov-Smirnov test of the consistency of two distributions. $S$ is a measure of the consistency of the observed hit pattern with the hypothesis of constant light emission by a muon. The simplest definition of the smoothness $S$ is $S=S_{j}^{\max }$, where $S_{j}^{\max }$ is that $S_{j}$, which has the largest absolute value, and $S_{j}$ is defined as

$$
S_{j} \equiv \frac{j-1}{N-1}-\frac{l_{j}}{l_{N}} .
$$

$l_{j}$ is the distance along the track between the points of closest approach of the track to the first and the $j^{\text {th }}$ hit module, with the hits taken in order of their projected position on the track. $N$ is the total number of hits. Tracks with hits clustered at the beginning or end of the track have $S$ approaching +1 or -1 , respectively. High quality tracks with $S$ close to zero, have hits equally spaced along the track. A graphical representation of the smoothness construction can be found in [30].

Extensions of this smoothness parameter include the restriction of the calculation to direct hits only or using the distribution of hit times $t_{i}$ instead of the distances $l_{i}$.

A particularly important extension is $S^{P^{\text {hit }}}$. In order to account for the granularity and asymmetric geometry of the detector one can replace the above formulation with one that models the hit smoothness expectation for the actual geometry of the assumed muon track. This can be accomplished by using the hit probabilities of all $N_{\mathrm{OM}}$, the number of operational OMs, (ordered along the track) as weights: $S^{P^{\text {hit }}}=\max \left(S_{j}^{P^{\text {hit }}}\right)$ with

$$
S_{j}^{P^{\mathrm{hit}}} \equiv \frac{\sum_{i=1}^{j} \Lambda_{i}}{\sum_{i=1}^{N_{\mathrm{OM}}} \Lambda_{i}}-\frac{\sum_{i=1}^{j} P^{\mathrm{hit}, \mathrm{i}}}{\sum_{i=1}^{N_{\mathrm{OM}}} P^{\mathrm{hit}, \mathrm{i}}} .
$$

$\Lambda_{i}=1$, if the OM $i$ was hit and 0 otherwise and $P_{\text {hit,i }}$ is the probability for $\mathrm{OM} i$ to be hit given the reconstructed track. The hit probabilities are calculated according to the algorithm in section 3.2.3. Smoothness selections are very efficient against secondary cascades, stopping muons and coincident muons from independent air showers.

Interesting AMANDA events are analyzed with multiple reconstruction algorithms. An event is most likely to have been reconstructed correctly, if the different algorithms produce consistent results.

For two reconstructions with directions $\mathbf{e}_{1}$ and $\mathbf{e}_{2}$, the space angle between them is given by $\Psi=\arccos \left(\mathbf{e}_{1} \cdot \mathbf{e}_{2}\right)$, which should be reasonably small for successful reconstructions. This concept can be extended to multiple reconstructions and their angular deviations from the 
average direction. For $n$ different reconstructed directions, $\mathbf{e}_{i}$, the average reconstructed direction, $\mathbf{E}$, is given by $\mathbf{E}=\sum_{i}^{n} \mathbf{e}_{i} /\left|\sum_{i}^{n} \mathbf{e}_{i}\right|$. We can define the parameter

$$
\Psi_{w}=\left(\sum_{i}\left[\arccos \left(\mathbf{e}_{i} \cdot \mathbf{E}\right)\right]^{w}\right)^{1 / w}
$$

$\Psi_{1}$ describes the average space angle between the individual reconstructions and $\mathbf{E} . \Psi_{2}$ is a different parameter, which treats the deviations between $\mathbf{E}$ and the $\mathbf{e}_{i}$ as "errors" and adds them quadratically. Small values of $\Psi_{1}$ or $\Psi_{2}$ indicate consistent reconstruction results.

The $\Psi$ parameters are a mathematically correct consistency check only when comparing the results of uncorrelated reconstructions of the same intrinsic resolutions. This is not the case when comparing different AMANDA reconstructions. Irrespective of the validity of such an interpretation, $\Psi_{1}$ or $\Psi_{2}$ are very efficient selection criteria, especially against almost horizontal muons and wide muon bundles.

A few additional selection parameters are closely related to first guess methods. The ratio of the eigenvalues of the tensor of inertia (see section 4.4) are a measure of the sphericity of the event topology, which is an efficient selection parameter against cascade backgrounds. Tracks reconstructed as down-going by the dipole fit (see section 4.3) that have a nonnegligible dipole moment, $M_{D A} \equiv|\vec{M}|$, indicate coincident muons from independent air showers. Larger values of the line-fit speed $v_{\mathrm{LF}}$ (see section 4.2) are an indication for longer muon-like, smaller values for more spherical cascade-like events.

Finally, two approaches evaluate the "intrinsic resolution" or "stability" of the reconstruction of each event. One approach quantifies the sharpness of the minimum found by the minimizers in $-\log (\mathcal{L})$ by fitting a paraboloid to it. The fitted parameters can then be used to classify the sharpness of the minimum. The other approach splits an event into sub-events (for example, containing odd- vs. even-numbered hits) and reconstructs the sub-events. If the reconstructed directions of the sub-events are different, then the reconstruction of the full event has a larger uncertainty.

\subsection{Analysis Strategies}

Analyses that search for neutrino induced muons must cope with a large background of atmospheric muons. The optimal choice of reconstruction and selection criteria varies strongly with different expectations for the energy and angular distribution of the signal events. The goal is to optimize the signal efficiency over the background or noise (square root of the background) based on sets of signal and background data.

- The selection criteria for background sensitive variables may be adjusted individually such that a specified fraction of signal events pass. After these first level criteria are set, the adjustment is repeated until the desired background rejection is reached. Each 
iteration defines a "cut-level", which corresponds to data sets of increasing purity. This simple method is used to derive a defined set of selection parameters for the performance section 7. However, less efficient criteria are mixed with more efficient criteria, and correlations of the variables are not taken into account. Therefore, this method does not achieve the optimum signal efficiency.

- An improvement to this method has been demonstrated in an AMANDA point source analysis [46,47]. Here, a selection criterion is only applied to the most sensitive variable, and the most sensitive variable is determined at each cut level. An interesting aspect of this point source search is that the experimental data themselves can be used as a background sample, which reduces systematic uncertainties from the background simulation. The selection criteria are not optimized with respect to signal purity but with respect to an optimal significance of a possible signal.

- Another approach is to combine the selection parameters into a single selection parameter, called event quality. This can be done by rescaling and normalizing each of the selection parameters according to the cumulative distribution of the signal expectation. The AMANDA analyses of atmospheric neutrinos [30,48] used this technique.

- Additional approaches use discriminant analysis [49] or neural nets $[6,50,51]$ to optimize the efficiency while taking into account the correlations between selection criteria and their individual selectivity. However, both methods depend critically on a good agreement between experiment and simulation. These methods quantify the efficiency of each parameter by including and excluding it from the optimization procedure.

- The "CutEval" method finds the optimum combination of selection parameters and cut values by numerically maximizing a significance function, $\mathcal{Q}$. An example is $\mathcal{Q}=$ $S / \sqrt{B}$, where $S$ is the number of signal events, and $B$ is the number of background events after selection.

The implementation proceeds in several steps. First, the most efficient selection parameter, $\mathcal{C}_{1}$, is the parameter that individually maximizes $\mathcal{Q}$. The next parameter, $\mathcal{C}_{2}$, is the parameter that maximizes $\mathcal{Q}$ in conjunction with $\mathcal{C}_{1}$. More parameters are successively determined until the addition of a new parameter fails to improve $\mathcal{Q}$. This procedure takes correlations between the selection criteria into account. The final number of selection parameters is reduced to a minimum, while maximizing the efficiency. Next, the optimal selection for this combination parameters is computed as a function of a boundary condition (e.g. the maximum number of accepted background events). This boundary condition is also used to define a single quality parameter.

Such a formalized procedure has to be carefully monitored, e.g. to handle potentially un-simulated experimental effects. The CutEval procedure is monitored by defining different, complementary optimization functions $\mathcal{Q}$, which allow real and simulated data to be compared [30,52-55]. 


\section{Performance}

This section describes the performance of the reconstruction methods. It is based on illustrative data selections, and the actual performance of a dedicated analysis can be different. Unless noted otherwise, the data shown is from Monte Carlo simulations of atmospheric neutrinos for AMANDA-II.

\subsection{First Guess Algorithms}

Since the first guess algorithms are used as a starting point for the full reconstruction, they should provide a reasonable estimate of the track coordinates. Also, these algorithms are used as the basis of early level filtering, and therefore need to be sufficiently accurate for that purpose, i.e. they should at least reconstruct the events in the correct hemisphere.

\section{Table 1}

\begin{tabular}{|l|c|c|}
\hline reconstruction & atm. $\mu$ & atm. $\nu$ \\
\hline direct walk & $1.5 \%$ & $93 \%$ \\
line-fit & $4.8 \%$ & $85 \%$ \\
dipole algorithm & $16.8 \%$ & $78 \%$ \\
\hline
\end{tabular}

The atmospheric muon and atmospheric neutrino detection efficiencies for a selection at $\theta \geq 80^{\circ}$ for the first guess algorithms.

As an example, Table 1 gives the passing efficiencies with respect to the AMANDA-II trigger for atmospheric neutrinos (signal) and atmospheric muons (background) for the first guess methods (see section 4), after the selection of events with calculated zenith angles larger $80^{\circ}$. The direct walk algorithm gives the best background suppression and the highest atmospheric neutrino passing rate. Correspondingly, it also gives the best initial tracks to the likelihood reconstructions.

\subsection{Pointing accuracy of the Track Reconstruction}

The angular accuracy of the reconstruction can be expressed in terms of a point spread function, which is given by the space angle deviation $\Psi$ between the true and the reconstructed direction of a muon corrected for solid angle. The space angle deviation is a combined result of two effects: a systematic shift in the direction and a random spread around this shift. In a point source analysis, for example, it is possible to correct for systematic shifts and be limited by the point spread function alone [47].

The zenith and space angular deviations are shown in figures 8 and 9 . They are obtained by the reconstruction algorithms as used in AMANDA-B10. The same event selection is used 


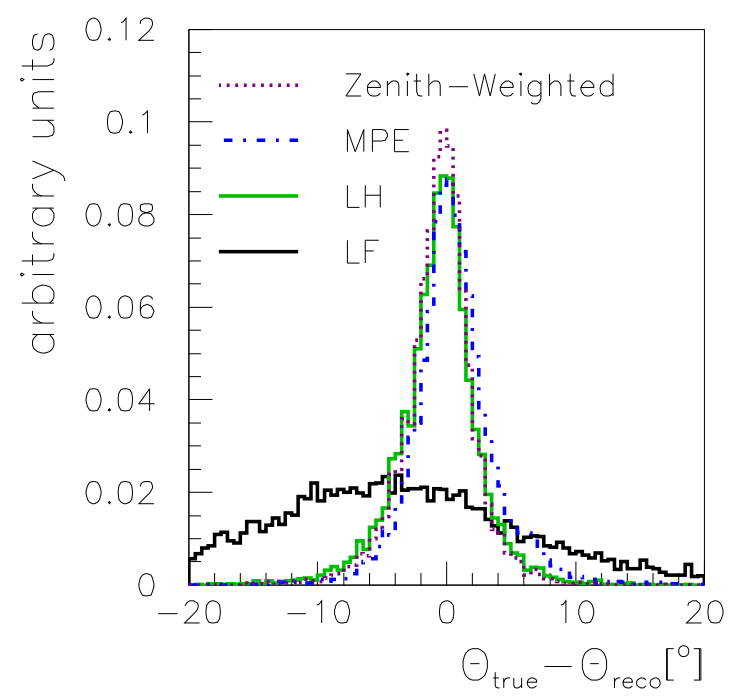

Figure 8. The zenith angle deviations for various reconstructions of AMANDA-B10. The result of an atmospheric neutrino simulation after the selection criteria of [30] is shown. The fits are a line-fit (LF), an iterated upandel fit (LH), an iterated zenith-weighted upandel fit and a MPE fit.

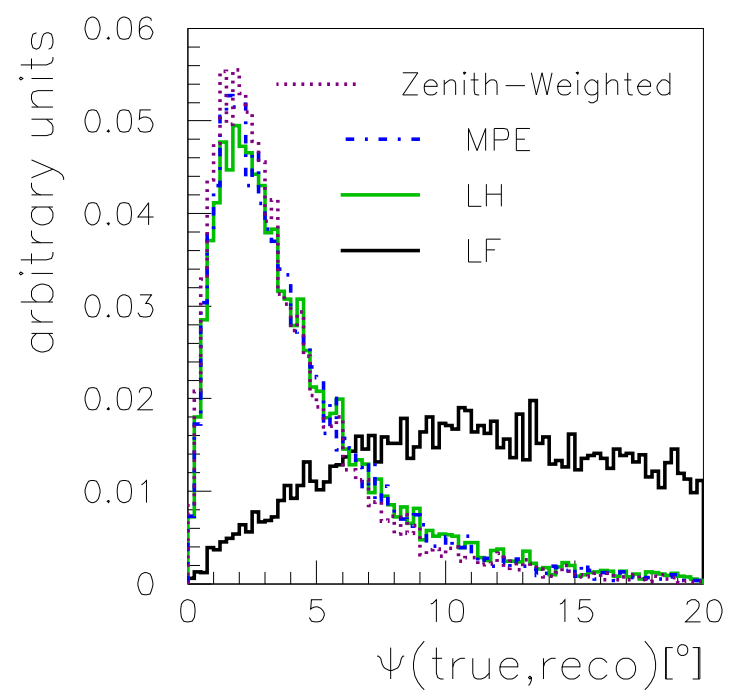

Figure 9. The distribution of space angle deviations for various reconstructions of AMANDA-B10. The result of an atmospheric neutrino simulation after the selection criteria of [30] is shown. The fits are a line-fit (LF), an iterated upandel fit (LH), an iterated zenith-weighted upandel fit and a MPE fit.

for all. As a general observation, the distributions of deviations for different reconstruction algorithms is surprisingly similar after a particular selection. Larger differences are usually seen in the selection efficiencies. A similar behavior is observed for AMANDA-II.

The dependence of the space angle deviation for the full AMANDA-II detector on the cut level ${ }^{6}$ for the $\mathrm{LH}$ reconstruction is shown in figure 10. The tighter the selection

$\overline{6}$ The cut levels defined here are typical and intended as demonstrating example. We use typical 


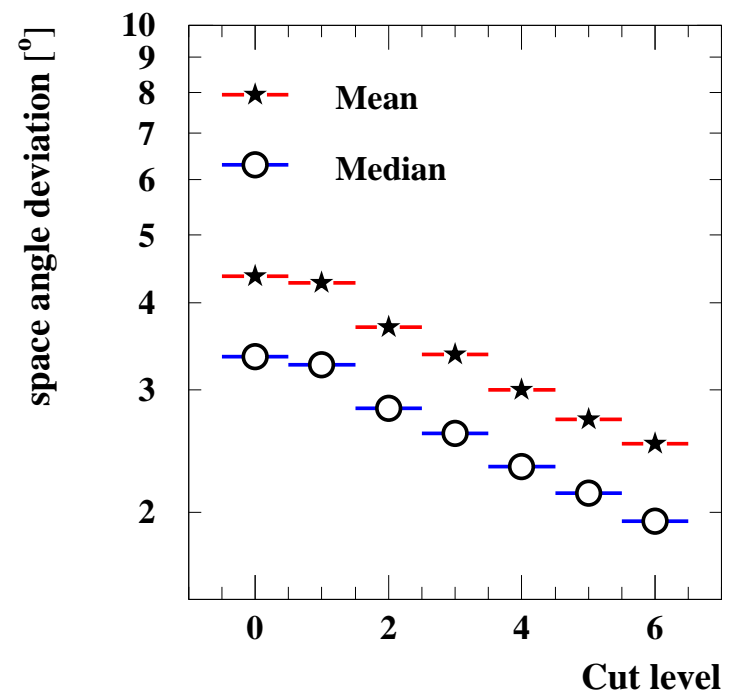

Figure 10. The dependence of the space angle deviation of the LH reconstruction in AMANDA-II on the event selection (cut levels).

criteria, the better the angular resolution. The same general trend is true for the other reconstructions. Tight criteria select events with unambiguous hit topologies, which are reconstructed better. The results for cut level 6 are shown in figures 11-13 as function of the energy and the zenith angle.

The angular resolution (see figure 11) has a weak energy dependence. The energy of the muon is taken at the point of its closest approach to the detector center. Best results are achieved for energies of $100 \mathrm{GeV}$ to $10 \mathrm{TeV}$. At energies $<100 \mathrm{GeV}$, the muons have paths shorter than the full detector, which limits the angular resolution. At energies $>10 \mathrm{TeV}$, more light is emitted due to individual stochastic energy loss processes along the muon track. Here, the hit pattern is not correctly described by the underlying reconstruction assumption of a bare muon track (see section 6.1).

The space angular resolution depends on the incident muon zenith angle (see figure 12). Again this is shown only for the LH reconstruction, the other reconstructions are similar. Up-going muons with $\cos \theta_{\mu} \simeq-0.7$ are best reconstructed, and horizontal muons are the worst, because of the geometry of the AMANDA-II detector. Nearly vertical events with $\cos \theta_{\mu} \simeq-1$ have a poorer angular resolution, because they illuminate fewer strings, which can cause ambiguities in the azimuth.

Systematic shifts also degrade the angular resolution. AMANDA observes a small zenith

selection parameters from section 6.2: the reconstructed zenith angle, $\theta^{\mathrm{DW}}>80^{\circ}, \theta^{\mathrm{LH}}>80^{\circ}$, $N_{\text {ch }}, N_{\text {dir }}^{\mathrm{LH}}(-15: 25), L_{\text {dir }}^{\mathrm{LH}}(-15: 75), \mathrm{L}^{\mathrm{LH}}, S^{\mathrm{LH}}$ and $\Psi_{1}(D W, L H, M P E)$. Our goal here is to illustrate the analysis, and we do not optimize with respect to efficiency and angular resolution. Instead each individual criterion is enforced in such a way that $95 \%$ of the events from the previous level would pass, and correlations between the parameters are ignored. Specific physics analyses will use selection criteria of higher efficiency and will achieve better angular resolutions than the $\simeq 2^{\circ}$, shown here. 


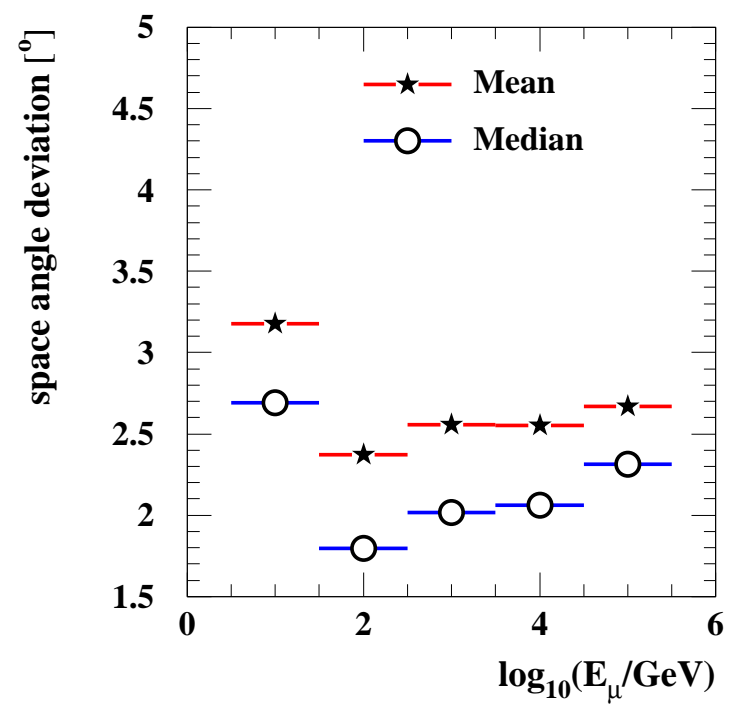

Figure 11. The dependence of the space angle deviation of the LH fit on the muon energy for AMANDA-II. Shown are mean (stars) and median (circles) for simulated atmospheric neutrinos.

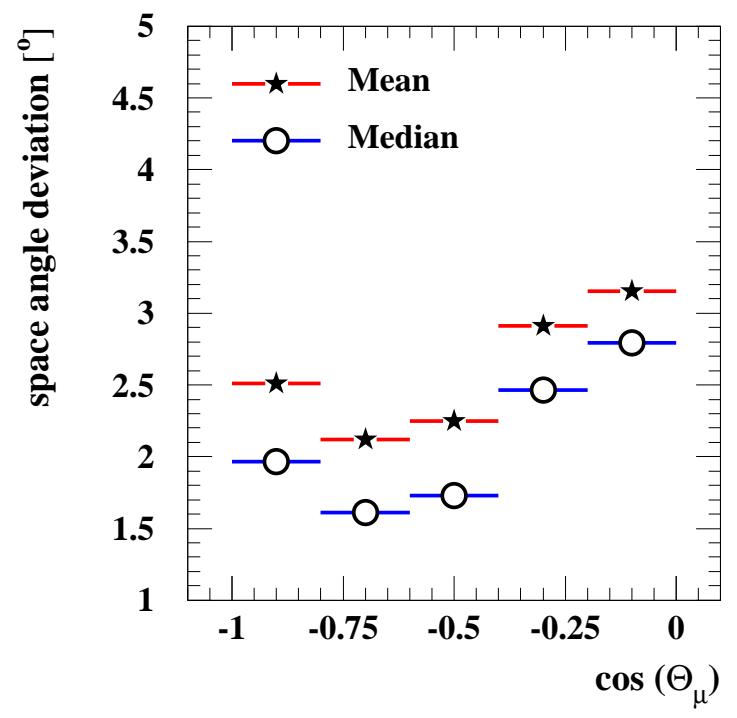

Figure 12. The space angle deviations of the LH fit as a function of the cosine of the incident zenith angle (for AMANDA-II). Shown are the mean (stars) and median (circles) for simulated atmospheric neutrinos.

dependent shift of the reconstructed zenith angle and no systematic shift in azimuth. This is shown in figure 13 for simulated atmospheric neutrinos in AMANDA-II. The size of this shift depends on the zenith angle itself, and it is determined by the geometry of AMANDA, which has a larger size in vertical than in horizontal directions. From a comparison with AMANDA-B10 data $[46,56]$, we observe that these shifts become smaller with a larger horizontal detector size. These shifts are confirmed by analyzing AMANDA events coincident with SPASE (see below).

These angular deviations have been obtained from Monte Carlo simulations. They can be 


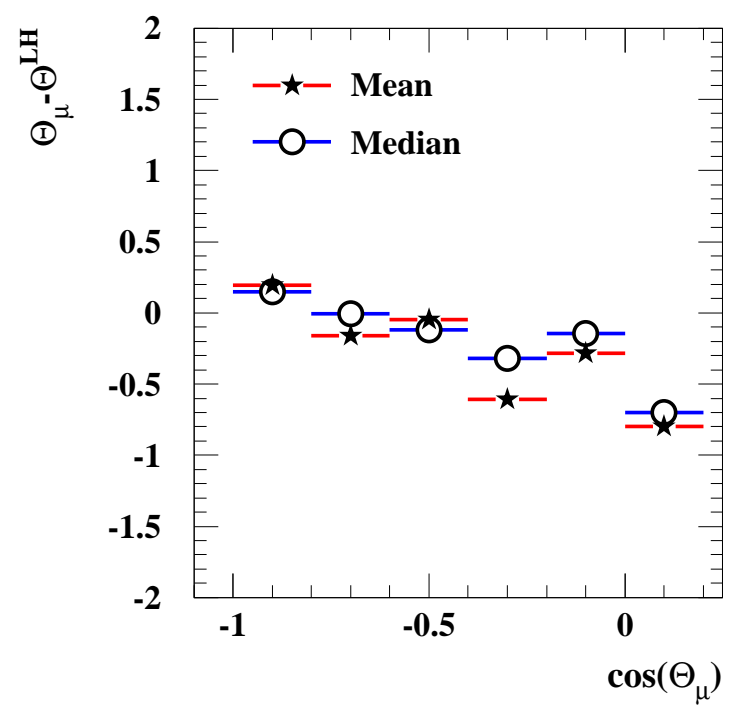

Figure 13. The zenith angle shift of the reconstruction versus the cosine of the incident angle. Shown are mean (stars) and median (circles) for simulated atmospheric neutrinos.

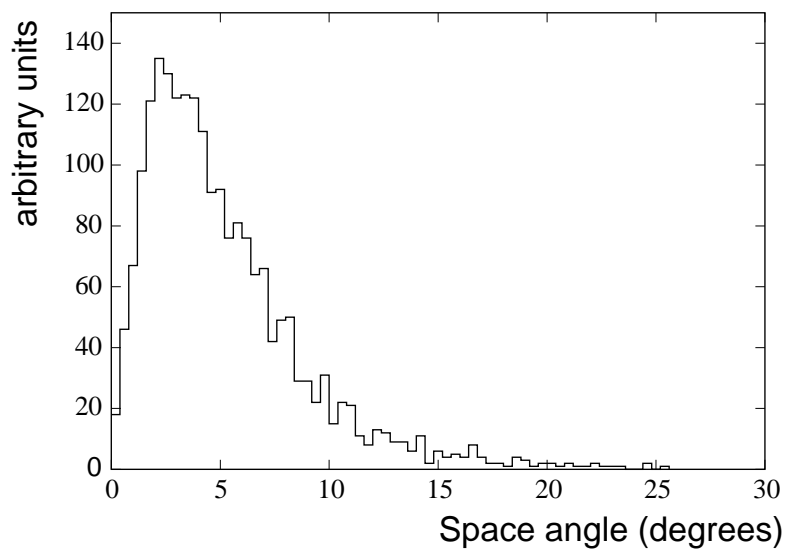

Figure 14. Distribution of the space angle deviations between air shower directions assigned by SPASE-2 and muon directions assigned by AMANDA-B10 for coincident events measured in 1997. The figure is not corrected for the systematic shift.

experimentally verified by analyzing coincident events between AMANDA and SPASE. An analysis of data from the 10 string AMANDA-B10 detector, shown in figure 14, confirms the estimate of $\simeq 3^{\circ}$ obtained from Monte Carlo studies for AMANDA-B10. Unfolding the estimated SPASE resolution of $\simeq 1^{\circ}$ confirms the estimated AMANDA-B10 resolution of $\simeq 3^{\circ}$ near the SPASE-AMANDA coincidence direction [8-10].

A simulation-independent estimate can be obtained by splitting the hits of individual events in two parts and reconstructing each sub-event separately. The difference in the two results gives an estimate of the total angular resolution. Such analyses are being performed at present and results will be published separately. 


\subsection{Energy Reconstruction}

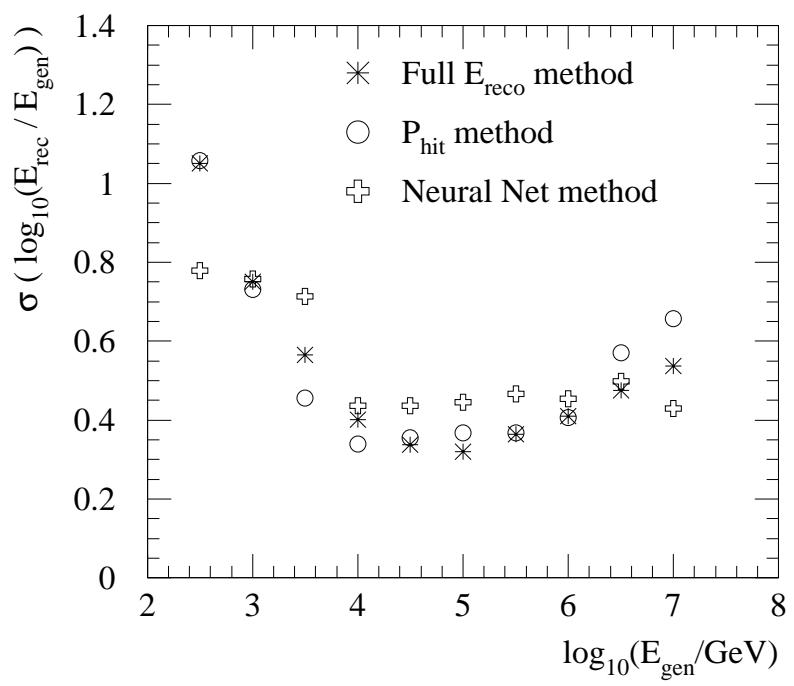

Figure 15. Comparison of the resolution of three different energy reconstruction approaches for AMANDA-B10. $E_{\text {gen }}$ is the generated energy (MC) and $E_{\text {rec }}$ the reconstructed energy.

The energy resolution of the three methods, described in section 3.2.4, is shown in figure 15 as function of the muon energy at its closest point to the AMANDA-B10 center. The resolution for AMANDA-B10 in $\Delta \log _{10} E$ is $\simeq 0.4$, for the interesting energy range of a few $\mathrm{TeV}$ to $1 \mathrm{PeV}$. Below $\simeq 600 \mathrm{GeV}$ the energy resolution is limited, because the amount of light emitted by a muon is only weakly dependent on its energy. Above $1 \mathrm{TeV}$ the resolution improves because radiative energy losses become dominant. Above $100 \mathrm{TeV}$ the resolution degrades, because energy loss fluctuations dominate.

Although these methods are quite different, their performances are similar. The full $E_{\text {reco }}$ and $P_{\text {hit }}$ methods achieve similar resolutions up to $1 \mathrm{PeV}$. The $P_{\text {hit }}$ method becomes worse above this energy, because in AMANDA-B10 almost all of the OMs are hit, and the method saturates. In contrast, the Neural Net method shows a slightly poorer resolution up to $1 \mathrm{PeV}$ but is better above. Its resolution is relatively constant over several decades of energy. This is an advantage when reconstructing an original energy spectrum with an unfolding procedure as in [36].

The AMANDA-II detector contains more than twice as many OMs as AMANDA-B10, and the energy resolution is better, especially at larger energies, $\sigma\left(\Delta \log _{10} E\right) \simeq 0.3$. The neural net reconstruction results for AMANDA-II are shown in figure 16. Finally, the recently installed transient waveform recorders (TWR) allow better amplitude measurements, which should significantly improve the results of the energy reconstructions, in particular, the full $E_{\text {reco }}$ method [57].

As discussed in section 1, the cascade channel can achieve substantially better resolutions, because the full energy is deposited inside or close of the detector. Energy resolutions in $\Delta \log _{10} E$ of $\leq 0.2$ and $\leq 0.15$ can be achieved by AMANDA-B10 and AMANDA-II, 


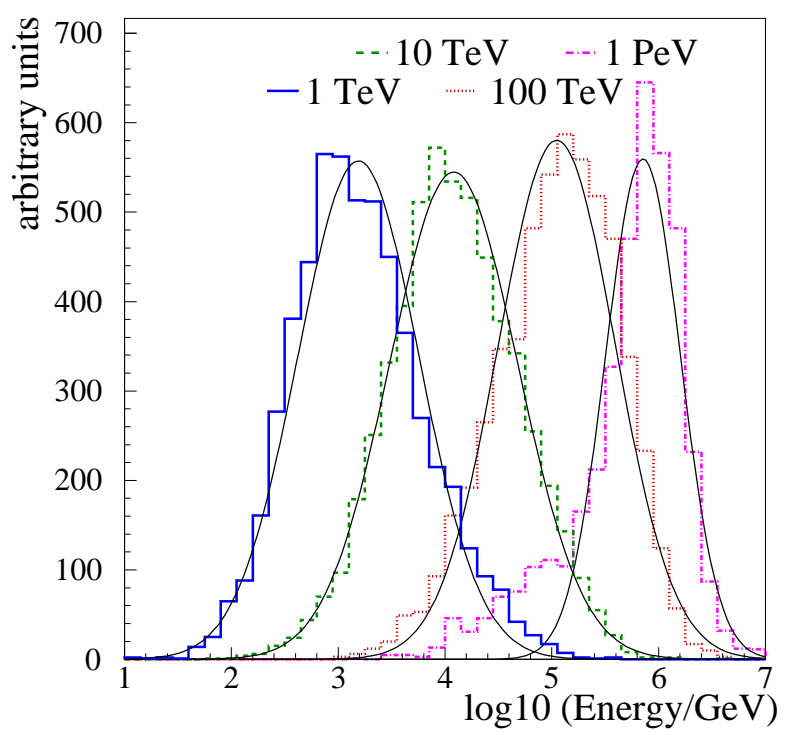

Figure 16. Energy reconstruction for simulated muons of different fixed energy in AMANDA-II, using the neural net method.

respectively [4].

\subsection{Systematic Uncertainties}

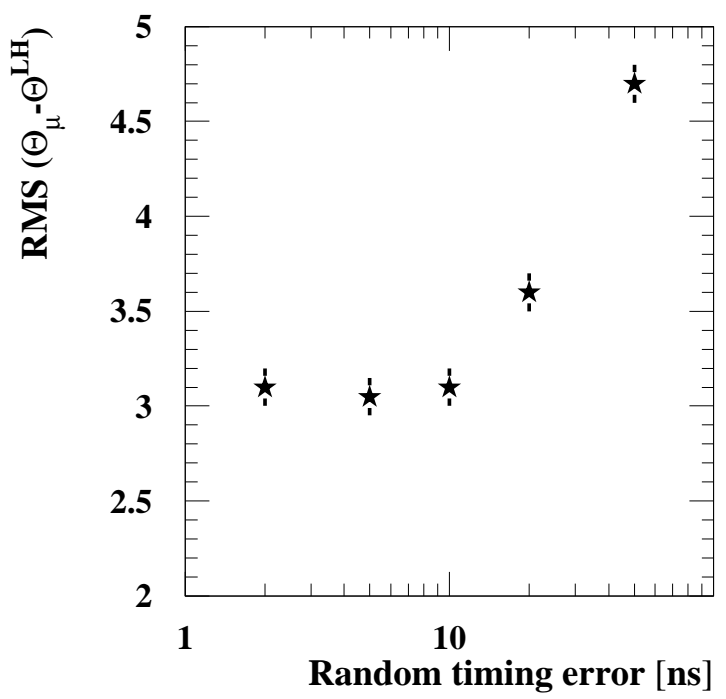

Figure 17. The zenith angle deviations (RMS) as function of an additional uncertainty in the $t_{0}$ time calibration. Data is shown for simulated atmospheric neutrino events in AMANDA-B10 with the selection of [30]. The transit time of the PMTs has been shifted without correcting for in the reconstruction. The shift is a fixed value for each PMT, obtained from a random Gaussian distribution.

Several parameters of the detector are calibrated and therefore only known with limited accuracy. These parameters include the time offsets, the OM positions and the absolute OM sensitivities. We have estimated the effects of these uncertainties on the resolution of 
AMANDA reconstructions [56]. As an example, figure 17 shows the effect of an additional contribution to the time calibration uncertainty for the 10 string AMANDA-B10 detector. The zenith angular resolutions for simulated atmospheric neutrino events only degrade when the additional timing uncertainties exceed 10 ns. Additional tests with similar results were done with non-random systematic shifts such as a depth dependent shift or a string dependent shift. Therefore, the angular resolution is insensitive to the uncertainties in the time calibration. The geometry of the detector is known to better than $30 \mathrm{~cm}$ horizontally and to better than $1 \mathrm{~m}$ vertically, which corresponds to timing uncertainties of $\lesssim 1$ or $3.5 \mathrm{~ns}$, respectively. Therefore, the geometry calibration is also sufficiently accurate.

Similarly, the effect of uncertainties on other parameters, like the absolute PMT efficiency, has been investigated. No indication was found that the remaining calibration uncertainties seriously affect the angular resolution or the systematic zenith angle offset. The combined calibration uncertainties are expected to affect the accuracy of the reconstruction by less than $5 \%$ in the zenith angle resolution and to less than $0.5^{\circ}$ in the absolute pointing offset.

\section{Discussion and Outlook}

We have developed methods to reconstruct and identify muons induced by neutrinos [30], inspite of the challenges of the natural environment and large backgrounds. These methods allow us to establish AMANDA as a working neutrino telescope. The reconstruction techniques described in this paper are still subject to improvement in several aspects:

The likelihood description: The likelihood functions for track reconstruction are based on the assumption of exactly one infinitely long muon track per event. Extensions of this model to encompass starting muon tracks (including the description of the hadronic vertex), stopping muons, muon bundles of non-negligible width, and multiple independent muons will be important, particularly in the context of larger detectors such as Ice Cube. Initial efforts fitting multiple muons with the direct walk algorithm have been useful in rejecting coincident down-going muons, and work toward reconstructing muon bundles has begun in the context of events coincident with SPASE air showers.

The p.d.f. calculation: The likelihood function is based on parametrizations of probability density functions (p.d.f.). The p.d.f. is obtained from Monte Carlo simulations, and its accuracy is limited by the accuracy of the simulation. Better simulations lead directly to a better p.d.f. and hence better reconstructions.

The p.d.f. parametrizations: The p.d.f. is parametrized by functions (e.g. the Pandel functions) which only approximate the full p.d.f.. More accurate parametrization functions will result in better reconstructions. For example, the scattering coefficient shows a significant depth dependence (see section 2). The current reconstruction is based on an average p.d.f. assuming depth-independent ice properties. While the track reconstruction is relatively insensitive to the accuracy of the parametrization, we expect a 
depth-dependent p.d.f. to have better energy reconstruction.

Complementary information: The current reconstruction algorithms do not include all available information in an event. In particular, correlations between detected PMT signals are ignored. For this reason dedicated selection parameters have been designed to exploit this information. They are used to discriminate between well reconstructed and poorly reconstructed events and improve the quality of the data sample. Future work will try to improve these parameters and expand the present likelihood description.

Transient waveform recorders: At the beginning of the year 2003, the detector readout has been upgraded with transient waveform recorders [57]. We expect a substantial improvement of the multiple-photon detection and the dynamic range in particular for high muon energies.

The construction of a much larger detector, the IceCube detector, will start in the year 2004. It will consist of 4800 PMT deployed on 80 vertical strings and will surround the AMANDA detector [58]. The performance of IceCube has been studied with realistic Monte Carlo simulations and similar analysis techniques as described in this paper [59]. The result is a substantially improved performance in terms of sensitivity and reconstruction accuracy. A direction accuracy of about $0.7^{\circ}$ (median) for energies above $1 \mathrm{TeV}$ is achieved. Similar to AMANDA, we expect a further improvement by exploiting the full information, avaliable from the recorded wave-forms, in the reconstruction.

Acknowledgments This research was supported by the following agencies: Deutsche Forschungsgemeinschaft (DFG); German Ministry for Education and Research; Knut and Alice Wallenberg Foundation, Sweden; Swedish Research Council; Swedish Natural Science Research Council; Fund for Scientific Research (FNRS-FWO), Flanders Institute to encourage scientific and technological research in industry (IWT), and Belgian Federal Office for Scientific, Technical and Cultural affairs (OSTC), Belgium. UC-Irvine AENEAS Supercomputer Facility; University of Wisconsin Alumni Research Foundation; U.S. National Science Foundation, Office of Polar Programs; U.S. National Science Foundation, Physics Division; U.S. Department of Energy; D.F. Cowen acknowledges the support of the NSF CAREER program. I. Taboada acknowledges the support of FVPI.

\section{References}

[1] E. Andrés, et al., Observation of High Energy Neutrinos with Cherenkov detectors embedded in deep Antarctic Ice, Nature 410 (6827) (2001) 441-443.

[2] J. G. Learned, K. Mannheim, High-Energy Neutrino Astrophysics, Annual Reviews of Nuclear and Particle Science 50 (2000) 679-749.

[3] J. G. Learned, S. Pakvasa, Detecting Nutau Oscillations at PeV Energies, Astroparticle Physics 3 (1995) 267-274. 
[4] J. Ahrens, et al., Search for Neutrino-Induced Cascades with the AMANDA Detector, Physical Review D 67 (2003) 012003, arXive:astro-ph/0206487.

[5] "Stephan Hundertmark, the AMANDA Collaboration, AMANDA-B10 Limit on UHE MuonNeutrinos, in: Proceedings of the 28th International Cosmic Ray Conference, Tsukuba, Japan, 2003.

[6] S. Hundertmark, et al., A Method to Detect UHE Neutrinos with AMANDA, in: Proceedings of the $27^{\text {th }}$ International Cosmic Ray Conference, Vol. 3, Hamburg, Germany, 2001, pp. 1129-1132, HE.236.

[7] J. E. Dickinson, et al., A new air-Cherenkov array at the South Pole, Nuclear Instruments and Methods in Physics Research A 440 (1) (2000) 95.

[8] J. Ahrens, et al., Calibration and Survey of AMANDA with the SPASE Detectors, accepted for publication in Astroparticle Physics.

URL http://www . amanda.uci.edu/documents.html

[9] X. Bai, et al., Calibration and Survey of AMANDA with SPASE, in: Proceedings of the $27^{\text {th }}$ International Cosmic Ray Conference, Vol. 3, Hamburg, Germany, 2001, pp. 977-980, HE.208.

[10] K. Rawlins, Measuring the Composition of Cosmic Rays with the SPASE and AMANDA Detectors, Ph.D. thesis, University of Wisconsin, Madison, Wi, USA (Oct. 2001). URL http://amanda. berkeley.edu/manuscripts/

[11] E. Andrés, et al., The AMANDA neutrino telescope: principle of operation and first results, Astroparticle Physics 13 (1) (2000) 1-20, arXive:astro-ph/9906203.

[12] D. F. Cowen, K. Hanson, the AMANDA Collaboration, Time Calibration of the AMANDA Neutrino Telescope with Cosmic Ray Muons, in: Proceedings of the $27^{\text {th }}$ International Cosmic Ray Conference, Vol. 3, Hamburg, Germany, 2001, pp. 1133-1136, HE.237.

[13] K. Woschnagg, et al., Optical Properties of South Pole Ice at Depths from 140 to 2300 Meters, in: D. Kieda, M. Salamon, B. Dingus (Eds.), Proceedings of the $26^{\text {th }}$ International Cosmic Ray Conference, Vol. 2, Salt Lake City, USA, 1999, pp. 200-203, HE.4.1.15.

[14] P. B. Price, K. Woschnagg, Role of Group and Phase Velocity in High-Energy Neutrino Observatories, Astroparticle Physics 15 (1) (2001) 97-100, arXive:hep-ex/0008001.

[15] K. Woschnagg, P. B. Price, Temperature Dependence of Absorption in Ice at $532 \mathrm{~nm}$, Applied Optics 40 (15) (2001) 2496-2500.

[16] P. B. Price, K. Woschnagg, D. Chirkin, Age vs depth of glacial ice at South Pole, Geophysical Research Letters 27 (13).

[17] Y. D. He, P. B. Price, Remote Sensing of Dust in Deep Ice at the South Pole, Journal Geophysical Research D 103 (14) (1998) 17041-17056.

[18] P. B. Price, L. Bergstöm, Optical Properties of Deep Ice at the South Pole: scattering, Applied Optics 36 (18) (1997) 4181-4194.

[19] P. Askebjer, et al., Optical Properties of Deep Ice at the South Pole: Absorption, Applied Optics 36 (18) (1997) 4168-4180. 
[20] Particle Data Group, Review of Particle Properties, European Physical Journal C 15 (1-4).

[21] L. A. Kuzmichev, On the Velocity of Light Signals in the Deep Underwater Neutrino Experiments, e-preprint, Moscow State University, arXive:hep-ex/0005036 (Mar. 2000).

[22] C. Spiering, et al., Track Reconstruction and Background Rejection in the Baikal Neutrino Telescope, in: Proceedings of the 3rd Nestor International Workshop, Pylos, Greece, 1993, p. 234, DESY 94-050.

[23] E. Carmona, Reconstruction Methods for the Antares Neutrino Telescope, in: Proceedings of the $2^{\text {nd }}$ Workshop on Methodical Aspects of Underwater/Ice Neutrino Telescopes, Hamburg, Germany, 2001, p. 111.

[24] A. Bouchta, Muon Analysis with the AMANDA-B four-string detector, Ph.D. thesis, Stockholms Universitet, Stockholm, Sweden, uSIP Report 1998-07 (1998).

URL http://amanda. berkeley .edu/manuscripts/

[25] R. A. Porrata, The Energy Spectrum of Pointlike Events in AMANDA-A, Ph.D. thesis, University of California, Irvine, Ca, USA (1997).

[26] P. Miočinović, Muon energy reconstruction in the Antarctic Muon and Neutrino Detector Array (AMANDA), Ph.D. thesis, University of California, Berkeley, Ca, USA (Dec. 2001). URL http://amanda . berkeley.edu/manuscripts/

[27] G. C. Hill, Bayesian event reconstruction and background rejection in neutrino detectors, in: Proceedings of the $27^{\text {th }}$ International Cosmic Ray Conference, Vol. 3, Hamburg, Germany, 2001, pp. 1279-1282, HE.267.

[28] Ty R. DeYoung and Gary C. Hill, et al., Application of Bayes' Theorem to Muon Track Reconstruction in AMANDA, in: Proceedings Advanced Statistical Techniques in Particle Physics, Durham, UK, 2002.

[29] R. D. Cousins, Conference summary talk, in: Proceedings Advanced Statistical Techniques in Particle Physics, Durham, UK, 2002.

[30] J. Ahrens, et al., Observation of High Energy Atmospheric Neutrinos with the Antarctic Muon and Neutrino Detector Array, Physical Review D 66 (1) (2002) 012005, arXive:astro-ph/0205109.

[31] T. R. DeYoung, Observation of Atmospheric Muon Neutrinos with AMANDA, Ph.D. thesis, University of Wisconsin at Madison, Madison, Wisconsin, USA (May 2001).

URL http://amanda . berkeley .edu/manuscripts/

[32] V. M. Aynutdinov, et al., Physics of Particles and Nuclei Letters 109 (2001) 43.

[33] A. Karle, Monte Carlo simulation of photon transport and detection in deep ice: muons and cascades, in: Proceedings of Workshop on the Simulation and Analysis Methods for Large Neutrino Telescopes, DESY-Proc-1999-01, DESY Zeuthen, Germany, 1999, pp. 174-185.

[34] C. H. Wiebusch, Muon reconstruction with AMANDA, in: Proceedings of Workshop on the Simulation and Analysis Methods for Large Neutrino Telescopes, DESY-Proc-1999-01, DESY Zeuthen, Germany, 1999, pp. 302-316. 
[35] D. Pandel, Bestimmung von Wasser- und Detektorparametern und Rekonstruktion von Myonen bis $100 \mathrm{TeV}$ mit dem Baikal-Neutrinoteleskop NT-72, Diploma thesis, HumboldtUniversität zu Berlin, Berlin, Germany (Feb. 1996).

URL http://www-zeuthen.desy.de/nuastro/publications/diploma/

[36] H. Geenen, Energy reconstruction and spectral unfolding of atmospheric leptons with the AMANDA detector, Diploma thesis, University of Wuppertal, Wuppertal, Germany (Nov. 2002).

URL http://amanda. berkeley .edu/manuscripts

[37] J. Ahrens, et al., Limits on diffuse fluxes of high energy extraterrestrial neutrinos with the AMANDA-B10 detector, Physical Review Letters 90 (2003) 251101, arXive:astro-ph/0303218.

[38] V. J. Stenger, Track fitting for DUMAND-II Octagon Array, External Report HDC-1-90, University of Hawai'i at Manoa, Manoa, Hawaii, USA (1990).

[39] CERN Information Technology Division, "PAW - Physics Analysis Workstation; User's Guide", CERN Program Library Long Writeup Q121, Geneva, Switzerland (1999).

[40] J. Jacobsen, C. Wiebusch, An Overview of Offline Software for AMANDA, in: Proceedings of Workshop on the Simulation and Analysis Methods for Large Neutrino Telescopes, DESYProc-1999-01, DESY Zeuthen, Germany, 1999, pp. 194-204.

[41] O. Streicher, C. Wiebusch, "recoos", muon and neutrino reconstruction for underwater/ice Cherenkov telescopes (2001).

URL http://www .ifh.de/nuastro/software/siegmund/

[42] O. Streicher, C. Wiebusch, "rdmc", a library for processing neutrino telescope data and MC files (2001).

URL http://www.ifh.de/nuastro/software/siegmund/

[43] G. C. Hill, S. Hundertmark, M. Kowalski, P. Miocinovic, T. Neunhöfer, P. Niessen, P. Steffen, O. Streicher, C. Wiebusch, "The SiEGMuND software package" (2001).

URL http://www.ifh.de/nuastro/software/siegmund/

[44] W. H. Press, S. A. Teukolsky, W. V. Vetterling, B. P. Flannery, "Numerical Recipies in C - The Art of Scientific Computing", 2nd Edition, Cambridge University Press, Cambridge, UK, 1997.

URL http://www.nr.com/

[45] F. James, the CERN Computing and Networks Division, "MINUIT - Function Minimization and Error Analysis; Reference Manual", CERN Program Library Long Writeup D506, Geneva, Switzerland, 94th Edition (1994).

[46] S. Young, A Search for Point Sources of High Energy Neutrinos with the AMANDA-B10 Neutrino Telescope, Ph.D. thesis, University of California at Irvine, Irvine, California, USA (Jul. 2001).

URL http://amanda.berkeley.edu/manuscripts/

[47] J. Ahrens, et al., Search for Point Sources of High Energy Neutrinos with AMANDA, Astrophysical Journal 583 (2003) 1040, arXive:astro-ph/0208006. 
[48] S. Böser, Separation of atmospheric neutrinos with the AMANDA-II detector, Diploma thesis, Technische Universität München, Munich, Germany (Apr. 2002).

[49] J. Ahrens, et al., Limits to the muon flux from WIMP annihilation in the center of the Earth with the AMANDA detector, Physical Review D 66 (3) (2002) 032006, arXive:astro-ph/0202370.

[50] P. Niessen, C. Spiering, et al., Search for Relativistic Monopoles with the AMANDA Detector, in: Proceedings of the $27^{\text {th }}$ International Cosmic Ray Conference, Vol. 4, Hamburg, Germany, 2001, pp. 1496-1498, HE.315.

[51] A. Biron, On the Rejection of Atmospheric Muons in the AMANDA Detector, Diploma thesis, Humboldt-Universität zu Berlin, Berlin, Germany, DESY-THESIS-1998-014 (Mar. 1998).

URL http://amanda.berkeley .edu/manuscripts/

[52] M. Gaug, AMANDA event reconstruction and cut evaluation methods, in: R. Wischnewski (Ed.), Proceedings of the $2^{\text {nd }}$ Workshop on Methodical Aspects of Underwater/Ice Neutrino Telescopes, Hamburg, Germany, 2001, p. 123.

[53] M. Gaug, Detection of Atmospheric Muon Neutrinos with the AMANDA Neutrino Telescope, Diploma thesis, Humboldt-Universität zu Berlin, Berlin, Germany (Oct. 2000).

URL http://amanda . berkeley.edu/manuscripts/

[54] M. Gaug, CutEval, Website, including manual (2000).

URL http://www-zeuthen.desy.de/\$\sim\$gaug/cuteval/

[55] M. J. Leuthold, Search for Cosmic High Energy Neutrinos with the AMANDA-B10 Detector, Ph.D. thesis, Humboldt-Universität zu Berlin, Berlin, Germany (Sep. 2001).

[56] A. Biron, Search for Atmospheric Muon-Neutrinos and Extraterrestric Neutrino Point Sources in the 1997 AMANDA-B10 Data, Ph.D. thesis, Humboldt-Universität zu Berlin, Berlin, Germany (Jan. 2002).

URL http://amanda. berkeley.edu/manuscripts/

[57] Wolfgang Wagner, the AMANDA Collaboration, New Capabilities of the AMANDA-II High Energy Neutrino Telescope, in: Proceedings of the 28th International Cosmic Ray Conference, Tsukuba, Japan, 2003.

[58] Shigeru Yoshida, the IceCube Collaboration, The IceCube High Energy Neutrino Telescope, in: Proceedings of the 28th International Cosmic Ray Conference, Tsukuba, Japan, 2003.

[59] J. Ahrens, et al., Sensitivity of the IceCube Detector to Astrophysical Sources of High Energy Muon Neutrinos, submitted for publication to Astroparticle Physics, arXive:astro-ph/0305196 (May 2003). 